Алгебра и анализ

Том. 18 (2006), № 3
St. Petersburg Math. J.

Vol. 18 (2007), No. 3, Pages 347-372

S 1061-0022(07)00954-5

Article electronically published on April 10, 2007

\title{
GENNADII MIKHAĬLOVICH GOLUZIN AND GEOMETRIC FUNCTION THEORY
}

\author{
G. V. KUZ'MINA
}

Dedicated to the 100th anniversary of Gennadiu Mikhailovich Goluzin's birth

\begin{abstract}
G. M. Goluzin crucially influenced the development and extension of geometric function theory. His results received world-wide recognition, and his monograph "Geometric theory of functions of a complex variable" has been a reference book for several generations of analysts.

This paper is a survey of Goluzin's scientific work on the occasion of the 100th anniversary of his birth.
\end{abstract}

\section{§1. Dates of G. M. Goluzin's Life And Work}

Gennadiu Mikhaylovich Goluzin was born January 24, 1906, in the town of Torzhok (nowadays, in the Tver' region) in a family of a railwayman. In 1924, he entered the Faculty of Mathematics of Leningrad State University, from which he graduated in 1929. In the same year, Goluzin's diploma work was published in "Matematicheskiı Sbornik". After graduation from the University and until the end of his life, Goluzin taught at the University. In 1936, when he defended his Candidate dissertation, the Scientific Council bypassed the Candidate degree and awarded him a higher academic degree of Doctor of physico-mathematical sciences. From 1939 on, Goluzin was the Chairman of the division of the theory of functions of a complex variable in Leningrad State University.

Goluzin loved teaching and taught with great energy. For a number of years, he taught the course of mathematical analysis for students specializing in mathematics, as well as a number of special courses, and directed a seminar on the geometric theory of functions of a complex variable. Goluzin's lectures were always very well structured and very well presented. Many scientists grew up in Goluzin's seminars, his students including Yu. E. Alenitsyn, S. A. Gel'fer, L. I. Kolbina, N. A. Lebedev, and I. M. Milin. Goluzin was an exceptionally modest and kind person, which made his contacts with students and colleagues informal and fruitful.

From the moment of foundation of the Leningrad Branch of the Mathematical Institute of the Academy of Sciences of the USSR in 1940, Goluzin simultaneously worked in this institute, too.

For his scientific merits, Goluzin was awarded the University First Prize for the year of 1946 and the State Prize for the year of 1947.

A result of Goluzin's scientific and pedagogical activity is his monograph "The geometric theory of functions of a complex variable", which was first published in 1952. The second edition of the monograph, complemented by several of Goluzin's papers and by an extensive survey of up-to-date developments written by Leningrad mathematicians, was

2000 Mathematics Subject Classification. Primary 30-02, 30C55.

Key words and phrases. Univalent function, conformal mapping, geometric function theory, quadratic differential, method of the extremal metric, symmetrization method. 
published in 1966. Goluzin's monograph has been translated into German and English. At the time of printing of the first edition of the book, after a long and difficult illness, Gennadiu Mikhallovich Goluzin died on the 17th of January, 1952, at the age of 45.

Goluzin's results received world-wide recognition. The monographs by Jenkins [J4, Duren [Du, Pommerenke [Pom3], and other outstanding analysts are devoted to geometric function theory. The monographs contain numerous references to Goluzin's papers and citations of his results.

\section{§2. MAin PRINCIPLES OF CONFORMAL MAPPINGS}

2.1. At the present time, geometric function theory is part of mathematical analysis. The objects of study in this theory are general classes of functions defined on a planar domain or on a Riemann surface. We emphasize that geometric function theory regards these classes of functions first of all as classes of mappings, and, to a significantly smaller degree, studies questions of representing functions in analytic form. Univalent functions play the main role in geometric function theory. A regular or meromorphic function is said to be "univalent" if it determines a one-to-one or, as is customary to say, a univalent mapping. The classes of conformal mappings constituted by univalent mappings are the simplest ones from the viewpoint of their geometric properties. On the other hand, univalent mappings possess a number of important extremal properties in many general classes of conformal mappings.

Geometric function theory was started by the Riemann mapping theorem (of 1851), which asserts that each simply connected domain that does not have a one-point boundary is univalently and conformally mapped onto the disk, and by various generalizations of the theorem to the case of multiply connected domains. In the one hundred and fifty years that have passed from the moment of appearance of the Riemann mapping theorem, geometric function theory has become a highly developed science having extensive links with related disciplines, such as mathematical physics, the theory of elasticity, aeroand hydromechanics, electro- and magnetostatics, and so on. The methods of geometric function theory are marked by great diversity. The main methods of geometric function theory are as follows: the area method, the parametric Loewner method, the variational method, the method of the extremal metric, and the symmetrization method. All of these methods are geometric in essence. The modern state of geometric function theory is discussed in $\S \S 3-5$ of the present survey.

Albert Baernstein excellently characterized the originality and beauty of geometric function theory in one of his papers ( $D$ is the domain of a univalent function $f$ ):

"The geometric properties of $f(D)$ and the analytic properties of $f$ are forever playing as cat and mouse. Some days one is cat, some days the other. We join forces with cat in hopes of together catching mouse. It is this ever shifting geometric-analytic to-andfro which makes univalent function theory one of the most fascinating of mathematical subjects."

2.2. Gennadiu Goluzin's work is a classic of geometric function theory; he enriched it with new approaches and results. He also played a crucial role in the development and extension of the theory of conformal mapping. Goluzin's special courses at Leningrad University contained many general results on the theory of conformal mappings of simply connected and multiply connected domains. Later, he included the results into his monograph "The geometric theory of functions of a complex variable".

In the monograph, Goluzin presented principles of univalent conformal mapping of simply connected domains: the Riemann mapping theorem, Koebe's results on the correspondence between boundaries, and the Carathéodory theorem on the convergence of a sequence of domains. The principles of conformal mapping of multiply connected 
domains contain proofs of theorems asserting that a multiply connected domain is univalently and conformally mapped onto canonical domains, in particular, onto the plane with parallel rectilinear cuts having prescribed slope. Furthermore, Goluzin presented a solution of the Dirichlet problem and properties of the Green function for a multiply connected domain. He also proved theorems on the existence of conformal mappings of a multiply connected domain onto the disk and onto the $n$-sheeted disk.

Significant attention in Goluzin's monograph was paid to metric properties of closed sets in the plane (the theory of capacity and harmonic measure), to majorization principles (the principle of hyperbolic metric and Lindelöf's principle), and to applications of these principles.

A major separate subject of Goluzin's monograph consists of various questions related to boundary properties of functions analytic in the disk or inside a rectifiable contour. I. I. Privalov considered some of these questions. We observe that Goluzin held Privalov's results in high regard and recommended Privalov's well-known monograph to the students specializing in mathematics as additional reading for the course in the theory of functions of a complex variable.

The second edition of Goluzin's monograph was supplemented by a number of his papers. Among them I mention the papers "On $p$-valent functions" [1940] (see [L3] for the role of the results of that paper in the general theory of the area method) and "On a variational method in the theory of analytic functions" [1952.

A collection of Goluzin's results in a volume of "Trudy MIAN" is devoted to properties of analytic functions with bounded mean absolute value.

Goluzin always tried to be complete in his presentation of the results of the theory of conformal mapping that were known at the time. For instance, in addition to Grötzsch's theorem on mapping onto the plane along arcs of logarithmic spirals, Goluzin presented interesting inequalities for the functions realizing such a mapping, inequalities that were established in P. R. Garabedyan and M. Schiffer's paper of 1949 and which had been published not long before that.

Goluzin paid great attention to simplicity and completeness of arguments: Goluzin's proofs significantly simplify the original proofs of many theorems by H. Grötzsch, P. Koebe, and others. In some cases, Goluzin suggested quite novel proofs and indicated various generalizations. In the next subsection, we discuss one of such cases.

2.3. One of the main classes of univalent functions defined in a simply connected domain is the class $S$ of functions of the form

$$
w=f(z)=z+c_{2} z^{2}+c_{3} z^{3}+\cdots
$$

that are regular and univalent in the disk $U=\{z:|z|<1\}$. Koebe's classical theorem asserts that if $f(z) \in S$, then the domain $f(U)$ contains the disk $|w|<1 / 4$ (see $\S 3$ ). The following theorem extends this result significantly.

Theorem I. For $f(z) \in S$, fix $n$ rays emanating from the origin at equal angles to each other, and on each find a boundary point of the domain $f(U)$ (closest to $w=0$ ). Then at least one of the points lies at a distance of at least $(1 / 4)^{1 / n}$ from $w=0$.

This theorem was proved by M. A. Lavrent'ev and V. M. Shepelev, and also by E. Rengel. Goluzin gave another proof, in which he took the circular annulus $r<|z|<$ 1 , where $r$ is arbitrarily small, and subdivided its image under $f(z)$ into a system of quadrangles by a special system of arcs emanating from the origin $w=0$ and returning to the same point. After that, he applied Grötzsch's well-known lemmas. 
We give the necessary definitions, where we use the modern terminology. (In the definitions below, we mean, as usual, classes of rectifiable or locally rectifiable Jordan curves.)

Suppose $D$ is a (curvilinear) quadrangle conformally equivalent to a rectangle $R$ with opposite sides of lengths $a$ and $b$. The quantity $M=a / b$ is called the modulus of the domain $D$ with respect to the class of curves in $D$ that join the sides of $D$ corresponding to the sides of $R$ of length $a$.

Suppose $D$ is a doubly connected domain conformally equivalent to the circular annulus $R=\{z: r<|z|<R\}$. The quantity $M^{(1)}(D)=\frac{1}{2 \pi} \log (R / r)$ is called the modulus of the domain $D$ with respect to the class of curves in $D$ separating the boundary components of $D$. The quantity $M^{(2)}(D)=2 \pi / \log (R / r)$ is called the modulus of the domain $D$ with respect to the class of curves in $D$ that join the boundary components of $D$.

Theorem II (The first and second Grötzsch lemmas). Suppose $D$ is a doubly connected domain conformally equivalent to the annulus $K=\{z: r<|z|<R\}$, and let $M^{(j)}(D)$, $j=1,2$, be the moduli of $D$ defined above.

1) Suppose $D_{k}, k=1, \ldots, n$, are disjoint doubly connected domains lying in $D$ and separating the boundary components of $D$. We denote by $M^{(1)}\left(D_{k}\right)$ the modulus of the domain $D_{k}$ with respect to the class of curves separating the boundary components of $D_{k}$. Then we have

$$
\sum_{k=1}^{n} M^{(1)}\left(D_{k}\right) \leq M^{(1)}(D) .
$$

2) Suppose $D_{l}, l=1, \ldots, m$, are disjoint quadrangles lying in $D$ each of which has a pair of opposite sides on distinct boundary components of $D$. We denote by $M^{(2)}\left(D_{l}\right)$ the modulus of the quadrangle $D_{l}$ with respect to the class of curves that join the opposite sides of $D_{l}$ lying on the boundary components of $D$. Then we have

$$
\sum_{l=1}^{m} M^{(2)}\left(D_{l}\right) \leq M^{(2)}(D) .
$$

Equality in (2) occurs only in the case where the domains $D_{k}$ are obtained by splitting the domain $D$ along curves corresponding to the circles in the annulus $K$ that are concentric with the boundary circles of $K$.

Equality in (3) occurs only in the case where the quadrangles $D_{l}$ are obtained by splitting $D$ along curves corresponding to radial segments in $K$.

Grötzsch's lemmas form a basis of his strip method. Using this method, Grötzsch obtained a large number of deep results, both for simply connected and for multiply connected domains. The method was a basis for the method of the extremal metric. (The method is discussed in the concluding section of the present survey.) Grötzsch's lemmas are well-known results of the indicated method and have found numerous applications.

To a large extent, Goluzin's proof of the theorem on $\sqrt[n]{1 / 4}$ foreshadowed the arguments used in modern studies of the extremal decomposition problem.

Using the same approach, Goluzin proved the following covering theorem for segments.

Theorem III. If $f(z) \in S$, then the domain $f(U)$ contains $n$ rectilinear segments emanating from the origin $w=0$ at equal angles and having sum of lengths arbitrarily close to $n$.

G. M. Goluzin and A. F. Bermant also obtained other covering theorems for segments and areas, which are related to the results presented above. 
2.4. Almost all of Goluzin's work deals with geometric function theory. However, Goluzin's first studies [1933, 1934, 1934a were devoted to questions related to Carleman's formula and problems of mathematical physics.

In the conclusion of this section, we discuss a joint result of Goluzin and Krylov [1933, which has become widely popular. We mean the following formula, which recovers an analytic function $f(z)$ in the Hardy class $H^{1}$ on the unit disk from the trace of $f(z)$ on a subset $E$ of the unit circle (we assume that $E$ has positive length):

$$
f(z)=\lim _{n \rightarrow \infty} \frac{1}{Q^{n}(z)} \frac{1}{2 \pi i} \int_{E} \frac{f(t) Q^{n}(t)}{t-z} d t, \quad|z|<1,
$$

where $Q$ is an exterior function in the unit disk such that $|Q|=e$ a.e. on $E$, and $|Q|=1$ a.e. on $\{|z|=1\} \backslash E$.

In the modern literature, this formula is not quite justly called "Carleman's formula", although Torsten Carleman proved quite a particular and special case of the formula, in which it is by no means easy to guess the elegant and very general form given to it in Goluzin's and Krylov's paper 1933. Extensive literature is devoted to this formula, which should have been called the "Carleman-Goluzin-Krylov formula " (see, e.g., the monograph [Ai], and also the monograph $[\mathrm{P}$ of applied character). A number of studies are devoted to generalizations and analogs of the formula (CGK) in multidimensional complex analysis, and also in the theory of ill-posed problems of mathematical physics. In a number of papers, it has been studied how the character and rate of convergence as $n \rightarrow \infty$ of the integrals occurring in (CGK) depend on the subspace in $H^{1}$ containing the function $f(z)$. The formula (CGK) yields the shortest proof of the boundary uniqueness theorem for functions in the class $H^{1}$. A modification of the formula (CGK) leads to explicit constructions that prove the Szegö-Kolmogorov-Kreln theorem on the weighted approximation of arbitrary functions on the circle or on the line by functions with nonnegative spectrum; see BarKh.

We return to geometric function theory. Having no possibility of characterizing Goluzin's entire scientific legacy in the framework of the present paper, in the next two sections we restrict ourselves to his studies devoted to the parametric Loewner method and the variational method.

\section{§3. The Parametric Loewner method}

3.1. In the 1920s and 1930s, geometric function theory was a relatively young science. The systematic study of classes of univalent functions was started by Koebe's papers (1907-1909) on the correspondence between boundaries under a univalent conformal mapping. A number of initial results in geometric function theory were obtained by the area method. The method involves the simple argument that the image of a domain under a univalent conformal mapping leaves uncovered a set of nonnegative area. Along with the class $S$, a well-known object of study in geometric function theory is the class $\Sigma$ of functions of the form

$$
f(z)=z+\alpha_{0}+\alpha_{1} z^{-1}+\cdots
$$

that are meromorphic and univalent in $U^{*}=\{z:|z|>1\}$. In 1914, T. H. Gronwall proved the "fundamental area theorem in the class $\Sigma$ ", known as Gronwall's theorem. The Koebe function

$$
K(z)=z(1-z)^{-2}=z+2 z^{2}+3 z^{3}+\cdots
$$

belongs to the class $S$ and maps the unit disk $U$ onto the entire plane with a radial cut emanating from the point $-1 / 4$. The Koebe $1 / 4$-theorem asserts that the image of $U$ under a mapping $w=f(z)$ in the class $S$ covers the disk $|w|<1 / 4$, and the 
circle $|w|=1 / 4$ contains a point not in $f(U)$ only for functions obtained by rotating the Koebe function $K_{\epsilon}(z)=\epsilon^{-1} K(\epsilon z),|\epsilon|=1$. L. Bieberbach established a number of remarkable properties of the Koebe functions. For example, the Koebe functions realize the maximum and minimum of $|f(z)|$ and $\left|f^{\prime}(z)\right|$ in the class $S$ for any fixed $|z|=r$, $0<r<1$. Bieberbach also showed that in the class $S$ we have the inequality $\left|c_{2}\right| \leq 2$, and $\left|c_{2}\right|=2$ only for the functions $K_{\epsilon}(z),|\epsilon|=1$. In 1916, Bieberbach conjectured that in the class $S$ for all $n \geq 2$ we have the inequality

$$
\left|c_{n}\right| \leq n
$$

and equality $\left|c_{n}\right|=n$ occurs only for the functions $K_{\epsilon}(z),|\epsilon|=1$.

Bieberbach's conjecture remained unproved for almost 70 years. The conjecture, especially during the first period of its existence, immensely influenced the development of methods of geometric function theory.

The first nonelementary method of geometric function theory was the parametric Loewner method. Originally, this method concerned the class $S_{1}$ of functions $f(z)$ with $f(0)=0$ and $f^{\prime}(0)>0$ that are regular and univalent in the unit disk $U$ and satisfy the condition $|f(z)|<1$ in $U$. The classical Loewner theory employs the fact that each function in the class $S_{1}$ (in the class $S$ ) is approximated by the corresponding "slit mappings". We also denote by $S_{1}^{\prime}$ the set of functions in $S_{1}$ that map $U$ onto a simply connected domain obtained by cutting the unit disk $U_{w}$ along a finite number of Jordan arcs. We denote by $S^{\prime}$ the set of functions in $S$ that map $U$ onto a domain obtained by cutting $\mathbb{C}_{w}$ along a finite number of Jordan arcs.

Loewner obtained the following parametric representation of functions in $S_{1}^{\prime}$.

Theorem IV. Each function $f(z) \in S_{1}^{\prime}$ with $f^{\prime}(0)=e^{-t_{0}}\left(0 \leq t_{0}<\infty\right)$ has the form $f(z)=f\left(z, t_{0}\right)$, where $f(z, t), 0 \leq t \leq t_{0}$, is the solution to the differential equation

$$
\frac{\partial f(z, t)}{\partial t}=-f(z, t) \frac{1+k(t) f(z, t)}{1-k(t) f(z, t)}
$$

with the initial condition $f(z, 0)=z$, where the function $k(t),|k(t)|=1$, is continuous on $\left[0, t_{0}\right]$ with the possible exception of a finite number of discontinuity points of the first type.

Using his method, Loewner proved inequality (5) for $n=3$.

Goluzin played a crucial role in the development and extension of the parametric Loewner method. Goluzin used the method in order to derive main results of the theory of univalent functions in a uniform way. In the case of the class $S$, Loewner's method is based on the following limiting form of Loewner's theorem.

Theorem V. Each function $f(z) \in S^{\prime}$ has the form

$$
f(z)=\lim _{t \rightarrow \infty} e^{t} f(z, t),
$$

where $f(z, t)$ is regular in $U$ as a function of $z$ and obeys the conditions $|f(z, t)|<1$ in $U, f(0, t)=0$, and $f^{\prime}(0, t)>0$, while $f(z, t)$ as a function of $t$ is a solution to equation (6) satisfying the initial condition $f(z, 0)=z$. Here, the function $k(t),|k(t)|=$ 1 , is continuous on the interval $0 \leq t<\infty$ with the possible exception of a finite number of discontinuity points of the first type.

3.2. Using Loewner's method, Goluzin obtained sharp inequalities for $\{\arg f(z) / z\}$ and $\left\{\arg z f^{\prime}(z) / f(z)\right\}$ in the class $S$ on the circle $|z|=r, 0<r<1$. One of the new results obtained by Goluzin with the help of this method was the sharp form of the rotation theorem in the class $S$. 
Theorem VI. If $f(z) \in S$, then for $|z|=r, 0<r<1$, we have the following sharp inequalities:

$$
\begin{aligned}
& \left|\arg f^{\prime}(z)\right| \leq 4 \arcsin r, \quad r \leq 2^{-1 / 2}, \\
& \left|\arg f^{\prime}(z)\right| \leq \pi+\log \frac{r^{2}}{1-r^{2}}, \quad r \geq 2^{-1 / 2},
\end{aligned}
$$

where we consider the branch of $\arg f^{\prime}(z)$ with $\arg f^{\prime}(0)=0$.

Having established a number of interesting inequalities for the functions $f(z, t)$, Goluzin obtained general distortion theorems for functions in the classes $S$ and $\Sigma$. One of them is a sharp inequality for the sum

$$
\operatorname{Re} \sum_{k, l=1}^{n} \gamma_{k} \gamma_{l} \log \frac{f\left(z_{k}\right)-f\left(z_{l}\right)}{z_{k}-z_{l}}
$$

in the class $\Sigma$ for any points $z_{k}$ in the domain $U^{*}$ and any complex numbers $\gamma_{k}, k=$ $1, \ldots, n$. The result is discussed below in connection with an application of the variational method.

For a function $f(z) \in \Sigma$, we define the coefficients $\omega_{k, l}$ by the expansion

$$
\log \frac{f(z)-f(\zeta)}{z-\zeta}=\sum_{k, l=1} \omega_{k, l} z^{-k} \zeta^{-l}, \quad|z|>1, \quad|\zeta|>1 .
$$

The coefficients $\omega_{k, l}$ are called the Grunsky coefficients of the function $f(z)$.

An impressive application of inequalities for the function $f(z, t)$ is the proof of the following theorem.

Theorem VII. For any complex numbers $x_{k}(k=1, \ldots, n ; n \geq 1)$, we have the inequalities

$$
\left|\sum_{k, l=1}^{n} \omega_{k, l} x_{k}, x_{l}\right| \leq \sum_{k}^{n} \frac{\left|x_{k}\right|^{2}}{k}
$$

Inequalities (7) were first obtained by Grunsky with the help of the method of contour integration. The inequalities give a necessary and sufficient condition for the univalence of a function and are among the main results of geometric function theory.

Using Loewner's method, Goluzin obtained a number of sharp estimates for the initial coefficients in the classes $S$ and $\Sigma$. The structure of the $n$th coefficient body in the class $\Sigma$ is significantly more complicated than in the class $S$. Already in 1949, Goluzin showed that the class $\Sigma$ contains functions such that $\left|\alpha_{3}\right|<1 / 2+e^{-6}$ in the expansion (4). This was a counterexample to the conjecture saying that for $n \geq 3$ we have $\left|\alpha_{3}\right|<2 /(n+1)$ in the class $\Sigma$.

Lebedev [L2] obtained new results in the theory of Loewner's method, which allowed him to find the sets of values of the system

$$
\left\{\alpha, \log f\left(z_{0}\right) / z_{0}\right\},
$$

where $\alpha=f^{\prime}(0)$, in the class $S_{1}$ and the sets of values of the quantities

$$
\log \left\{z_{0} f^{\prime}\left(z_{0}\right) / f\left(z_{0}\right)\right\}, \quad \log \left\{\alpha z^{2} f^{\prime}\left(z_{0}\right) / f\left(z_{0}\right)^{2}\right\}, \quad \text { and } \quad \log \left\{\alpha f^{\prime}\left(z_{0}\right)\right\}
$$

in the class $S_{1}\left(\left|f\left(z_{0}\right)\right|\right)$ of functions in $S_{1}$ with fixed value of $\left|f\left(z_{0}\right)\right|$. We note that the results of [L2] were used in Dubinin's recent paper [D6] devoted to inequalities for algebraic polynomials. 
3.3. Goluzin's studies were continued by P. P. Kufarev and other authors. A generalization of Loewner's equation is the Loewner-Kufarev equation

$$
\frac{d w}{d t}=-w P(w, t),
$$

where the function $P(w, t)$ is defined for $w \in U$ and $t \in[0, \infty)$. It belongs to the Carathéodory class of functions with positive real part for each $t>0$ and is measurable with respect to $t$ for each $w \in U$. Kufarev obtained the following sufficient criterion for a function to belong to the class $S$.

Theorem VIII. Suppose that a function $P(w, t)$ satisfies the conditions indicated above and is integrable on the segment $0 \leq t \leq T<\infty$ for each $w \in U$. Then equation (8) has a unique solution $w=f(z, t), 0 \leq t<\infty$, satisfying the initial condition $f(z, 0)=z$. The function $e^{t} f(z, t)$ belongs to the class $S$ for each fixed $t$ and uniformly converges as $t \rightarrow \infty$ to a function $f(z) \in S$ on each closed subset of the unit disk $U$.

Ch. Pommerenke and V. Ya. Gutlyanskiư logically completed the Loewner-Kufarev theory by showing that each function $f(z) \in S$ is represented via a solution of LoewnerKufarev's equation (8).

We observe that the classical Loewner theory has the advantage that Loewner's equation (6) is significantly simpler than equation (8) and has a simple geometric interpretation.

The results of the classical Loewner method and those of the variational method are related in the following way. General results of the variational method show that maps of the unit disk $U$ onto the plane cut along a finite number of analytic arcs are extremal for a large number of problems in the theory of univalent functions. Consequently, such mappings are represented via Loewner's equation (6) in terms of a unimodular function $k(t)$. This fact suggested using the differential equation for the conformal map obtained by the variational method on the one hand, and Loewner's equation on the other hand, in order to reduce finding the function $k(t)$ that occurs in Loewner's equation to a solution of a certain boundary problem for a system of differential equations. Schiffer indicated the possibility of such an approach in 1945 (see [Du]). Lebedev continued general consideration of this question (see AlKuzL). He started with Goluzin's variational formula. In Kufarev's papers, the indicated approach took a final form, and the method created by him became known as Kufarev's variational-geometric method. Using this method, members of the Tomsk mathematical school solved a number of difficult extremal problems; in this connection, see AlKuzL and Aleksandrov's book $[\mathrm{A}$.

V. V. Goryainov developed and applied the semigroup aspect of the Loewner-Kufarev theory. Concerning these questions, see [Kuz4], where an extensive bibliography can be found.

3.4. In 1984, Louis de Branges proved Bieberbach's conjecture. This proof turned out to be totally surprising and can be ranked among the most astonishing mathematical achievements of the 20th century. The Lebedev-Milin inequality plays an essential role in de Branges' proof. The inequality allows one to estimate the modulus of the $n$th coefficient $c_{n}$ of a function $f(z)$ in the class $S$ by a combination of the initial logarithmic coefficients of $f(z)$. The logarithmic coefficients $\gamma_{k}$ of $f(z)$ are defined by the expansion

$$
\log \frac{f(z)}{z}=\sum_{k=1}^{n} 2 \gamma_{k} z^{k} .
$$


The Lebedev-Milin inequality and the assertion on equality cases immediately imply that if for each function $f(z)$ in the class $S$ for all $n \geq 2$ we have

$$
\sum_{k=1}^{n}(n+1-k) k\left|\gamma_{k}\right|^{2} \leq \sum_{k=1}^{n} \frac{n+1-k}{k},
$$

then Bieberbach's conjecture is true. De Branges proved inequality (9): he established a general averaging inequality for the initial coefficients of bounded functions in the class $S$, from which inequality (9) follows by passage to a limit. The proof involves the classical Loewner theory for the class $S_{1}$ (see Theorem IV).

The original proof of Bieberbach's conjecture was published in the "LOMI Preprints" series; see Br1. After that, de Branges published a later presentation of his proof in $[\mathrm{Br} 2$. A somewhat revised version of de Branges' original proof was presented in the paper [FoKuz] by Kuz'mina and Fomenko, where the history of the proof was also described briefly.

FitzGerald and Pommerenke [FiPom] suggested a version of de Branges' proof, which is technically simpler, and where Loewner's theory is used directly for the class $S$.

\section{$\S 4$. VARIATIONAL METHOD}

4.1. The calculus of variations for univalent functions differs fundamentally from the classical calculus of variations due to a substantial nonlinearity of classes of univalent mappings. The boundary variational method created by Schiffer in 1938 was the first variational method of the theory of univalent functions that received wide acceptance. The method is based on the following lemma.

Theorem IX (Fundamental lemma of the boundary variational method). Suppose $\Gamma$ is a continuum on the sphere $\overline{\mathbb{C}}_{w}$. We assume that there exists an analytic function $s(w) \not \equiv 0$ on $\Gamma$ such that for each finite point $\omega_{0} \in \Gamma$ and every univalent function $V(w)$ representable in the form

$$
V(w)=w+A_{0}+A_{1} \rho^{2}\left(w-w_{0}\right)^{-1}+O\left(\rho^{3}\right)
$$

uniformly in an arbitrary closed subdomain $\left|w-w_{0}\right|>\rho$, we have the inequality

$$
\operatorname{Re}\left\{A_{1} \rho^{2} s\left(w_{0}\right)\right\}+O\left(\rho^{3}\right) \geq 0 .
$$

Then $\Gamma$ is an analytic curve and is represented parametrically via a function $w=w(t)$ satisfying the equation

$$
w^{\prime 2}(t) s[w(t)]+1=0, \quad w^{\prime}(t)=d w / d t .
$$

The proof involves the topological Haslam-Jones lemma, which implies that a continuum satisfying certain conditions is a rectilinear segment.

In a number of papers, the boundary variational method was later extended to the case of variations of a more special form. However, it is difficult to construct variations that allow one to consider classes of functions satisfying additional geometric conditions, which remains a disadvantage of the boundary variational method.

In 1943, Schiffer worked out an interior variational method, which is based on results of potential theory. Schiffer and his students obtained numerous applications of the method to extremal problems of geometric function theory. The indicated method was extended to the case of Riemann surfaces SSp.

In a series of papers of 1946-1951, Goluzin worked out his own version of the interior variational method and applied it to various extremal problems of geometric function theory. Goluzin's variational method is based on the following theorem. 
Theorem X. Suppose $w=f(z)$ is a function with $f(0)=0$ regular and univalent in the unit disk $U$. We assume that

$$
F(z, \lambda)=f(z)+\lambda q(z)+O\left(\lambda^{2}\right)
$$

is an analytic function of $z$ and $\lambda$ for $0<r \leq|z|<1$ and $|\lambda|<\lambda_{0}$ and that $F(z, \lambda)$ is univalent in the circular annulus $r \leq|z|<1$ for each fixed $\lambda, 0<\lambda<\lambda_{0}$. We denote by $D_{\lambda}$ the smallest (with respect to inclusion) simply connected domain containing the set of values of $F(z, \lambda)$ in the annulus $r \leq|z|<1$. If a function $f^{*}(z)$ maps the unit disk $U$ onto $D_{\lambda}$ univalently, then

$$
f^{*}(z)=f(z)+\lambda q(z)-\lambda z f^{\prime}(z) \overline{S(1 / \bar{z})}+O\left(\lambda^{2}\right) ;
$$

here $S(z)$ is the principal part of the Laurent series expansion of the function $q(z) /\left[z f^{\prime}(z)\right]$ in the annulus $r<|z|<1$.

In Theorem X, we can put

$$
F(z, \lambda)=f(z)+\lambda \sum_{k=1}^{n} \frac{A_{k} f^{2}(z)}{f(z)-w_{k}}, \quad w_{k} \in f(U), A_{k} \in \mathbb{C},
$$

which is convenient for a number of applications.

Goluzin's proof of Theorem X involved properties of majorizing series. Pommerenke Pom2 gave another proof of Theorem $X$, which is elementary, with the exception of an application of the Carathéodory theorem on the convergence of a sequence of domains to a kernel.

4.2. Using the variational method, Goluzin established a number of general results of distortion theorem type for univalent functions, where he completely answered the question about extremal mappings. Furthermore, when investigating extrema of certain quantities, he succeeded in finding their ranges. Here is one such result.

Theorem XI. Suppose $z_{1}, \ldots, z_{n}(n \geq 1)$ are $n$ distinct points in the domain $U^{*}$, and $\gamma_{1}, \ldots, \gamma_{n}$ are complex numbers. For functions $f(z) \in \Sigma$, we have the sharp inequality

$$
\operatorname{Re} \sum_{k, l=1}^{n} \gamma_{k} \gamma_{l} \log \frac{f\left(z_{k}\right)-f\left(z_{l}\right)}{z_{k}-z_{l}} \leq-\sum_{k, l=1}^{n} \gamma_{k} \overline{\gamma_{l}} \log \left(1-1 / z_{k} \overline{z_{l}}\right) .
$$

Equality in (10) occurs only for functions defined in the domain $U^{*}$ by the equation

$$
\sum_{k=1}^{n} \log \frac{f(z)-f\left(z_{k}\right)}{z-z_{k}}=-\sum_{k=1}^{n} \overline{\gamma_{k}} \log \left(1-1 / z \overline{z_{k}}\right) .
$$

These functions map the domain $U^{*}$ onto the entire $w$-plane cut along a curve satisfying the equation

$$
\operatorname{Re} \sum_{k=1}^{n} \gamma_{k} \log \left(w-f\left(z_{k}\right)\right)=\text { const. }
$$

Next, in the domain $U^{*}$ we have the inequality

$$
\left|\sum_{k, l=1}^{n} \gamma_{k} \gamma_{l} \log \frac{f\left(z_{k}\right)-f\left(z_{l}\right)}{z_{k}-z_{l}}\right| \leq-\sum_{k, l=1}^{n} \gamma_{k} \overline{\gamma_{l}} \log \left(1-1 / z_{k} \overline{z_{l}}\right)
$$

determining the set of values of the sum on the left-hand side. 
For $n=1$, estimate (10) implies the following theorem on the distortion of chords:

$$
\left|\log \frac{f(z)-f\left(z^{\prime}\right)}{z-z^{\prime}}\right| \leq-\log \left(1-r^{-2}\right), \quad|z|=\left|z^{\prime}\right|=r,
$$

and the following distortion theorem in the class $\Sigma$ :

$$
1-|z|^{-2} \leq\left|f^{\prime}(z)\right| \leq\left(1-|z|^{-2}\right)^{-1} .
$$

Theorems XI and VII had numerous generalizations and inspired the appearance of various inequalities of general type in classes of univalent mappings. The theorems were extended to the case of $n$-connected domains (see [G, M, KrKuh]) and quasiconformal mappings (see KrKuh $)$.

Using the same variational method, Goluzin obtained inequalities determining the sets of values of the quantities $f\left(z_{0}\right) / z_{0}$ and $z_{0} f^{\prime \prime}\left(z_{0}\right) / f^{\prime}\left(z_{0}\right)$ in the class $\Sigma$; these inequalities contain elliptic integrals of the first and the second kind.

Using Goluzin's variational method, Lebedev [L1] obtained a number of results on the set of values of the quantity

$$
\log \left\{z_{0}^{\lambda} f^{\prime}\left(z_{0}\right)^{1-\lambda} / f\left(z_{0}\right)^{\lambda}\right\}
$$

for $f$ ranging over $S$, where $\lambda$ is an arbitrary real parameter.

4.3. The method of extremal metric entered mathematics in the 1950s. Of all methods of geometric function theory, this one is most closely related to differential geometry and topology. It revealed the important role of quadratic differentials in extremal questions of the theory of univalent mappings. This role was discovered by O. Teichmüller.

A quadratic differential is an expression of the form $Q(z) d z^{2}$, where $Q(z)$ is a meromorphic function defined in a domain or on a Riemann surface. The curves on which we have $Q(z) d z>0$ are called the trajectories of $Q(z) d z$. The poles and zeros of $Q(z)$ are called the poles and zeros of the differential $Q(z) d z^{2}$. Starting with Grötzsch's results and with studies of his own, O. Teichmüller stated (without proof) a general principle describing how special features of an extremal problem determine singularities of the associated quadratic differential. Facts about the local and global structure of quadratic differentials are presented in [J4] and [Pom3, Chapter 8]; cf. [SchaSp]. Critical trajectories of the associated quadratic differential $Q(z) d z^{2}$ play a special role in the description of extremal configurations, the limit endpoints of the trajectories being the zeros and poles of $Q(z) d z^{2}$.

The theory of quadratic differentials not only has led to the final formulations of the results of the variational method, but it has also simplified their proofs significantly. For example, the "fundamental lemma of the boundary variational method" (see Theorem IX) immediately allows us to characterize extremal mappings in terms of critical trajectories of a quadratic differential. In the problems on the maximum of the Goluzin functional and on that of the Grunsky functional, the associated quadratic differentials are complete squares of rational functions, for which reason the extremal mappings of these theorems are described in terms of elementary functions.

Goluzin applied his variational method to investigating a number of questions of geometric function theory, which had been and would have remained open for a long time. One of them is N. G. Chebotarëv's variational problem on the continuum of the smallest capacity containing a given system of points. Suppose $a_{1}, \ldots, a_{n}$ is a system of distinct points on $\mathbb{C}$, and denote by $E\left(a_{1}, \ldots, a_{n}\right)$ a continuum of the smallest capacity containing the points. The problem on the smallest capacity continuum was studied by Grötzsch, who established the uniqueness of the extremal continuum with the help of his strip method. Lavrent'ev investigated this problem with the help of his variational-geometric 
method under the additional important assumption that the required continuum consists of analytic arcs. Goluzin obtained a final result for the question. In terms of the associated quadratic differential, this result is stated as follows.

Theorem XII. Suppose $a_{1}, \ldots, a_{n}(n \geq 3)$ is a system of distinct points of $\mathbb{C}$. The continuum $E\left(a_{1}, \ldots, a_{n}\right)$ is the union of the closures of critical trajectories of the quadratic differential

$$
-P(z)\left\{\prod_{k=1}^{n}\left(z-a_{k}\right)\right\}^{-1} d z^{2}
$$

where $P(z)=\left(z-c_{1}\right) \cdots\left(z-c_{n-2}\right)$ is a polynomial of degree $n-2$. We let $\zeta=g(z)$ be a conformal homeomorphism of the domain $D=\overline{\mathbb{C}} \backslash E$ onto $U^{*}$ with $g(\infty)=\infty$. The function $\zeta=g(z)$ satisfies in $D$ the equation

$$
\frac{d \zeta^{2}}{\zeta^{2}}=-Q(z) d z^{2}
$$

The zeros $c_{1}, \ldots, c_{n-2}$ of the polynomial $P(z)$ are determined from the condition that the closures of critical trajectories of the differential (11) have connected union.

Pirl $\mathrm{Pi}$ found analytic conditions determining the continuum $E\left(a_{1}, \ldots, a_{n}\right)$ and described geometric properties of $E\left(a_{1}, \ldots, a_{n}\right)$ in the case where $n=3$. If the points $a_{1}$, $a_{2}$, and $a_{3}$ do not lie on a line, then $E\left(a_{1}, a_{2}, a_{3}\right)$ consists of three analytic arcs meeting at a point $c_{1}=c_{1}\left(a_{1}, a_{2}, a_{3}\right)$, which is a unique zero of the differential (11).

The above theorem led to a complete solution of the problem on cap $E\left(a_{1}, a_{2}, a_{3}\right)$ : the result is stated as a system of equations for cap $E\left(a_{1}, a_{2}, a_{3}\right)$ and the zero $c_{1}\left(a_{1}, a_{2}, a_{3}\right)$ of the associated quadratic differential, the equations involving elliptic functions. If the triple of the points $a_{1}, a_{2}$, and $a_{3}$ is symmetric with respect to a line, then the problem reduces to considering the continuum $E\left(0, e^{i \alpha}, e^{-i \alpha}\right)$ for $0 \leq \alpha \leq \pi / 2$. In this case, the problem simplifies a great deal, which allows one to investigate the quantity cap $E\left(0, e^{i \alpha}, e^{-i \alpha}\right)$ as a function of $\alpha$.

In the general case, Jenkins [J5] investigated the dependence of cap $E\left(a_{1}, a_{2}, a_{3}\right)$ on the position of the points $a_{1}, a_{2}$, and $a_{3}$

Using the symmetrization method, Solynin [So1] and Emel'yanov [E] strengthened the result in [J5] as follows.

Theorem XIII. If a point a moves along an arc of an ellipse with foci at the points -1 and +1 so that $\arg a$ increases from 0 to $\pi / 2$, then cap $E(-1,1, a)$ decreases strictly monotonically.

The problem on cap $E\left(a_{1}, a_{2}, a_{3}\right)$ is related to a number of extremal problems of geometric function theory. For $n \geq 4$, the solution of the problem on cap $E\left(a_{1}, \ldots, a_{n}\right)$ is known only in certain symmetric cases (see, e.g., Fedorov's paper [F2]).

We discuss the problem on the maximum $d_{n}$ of the $n$th diameter,

$$
d_{n}(E)=\max _{c_{k}, c_{l} \in E}\left(\prod_{1 \leq k<l \leq n}\left|c_{k}-c_{l}\right|\right)^{2 /[n(n-1)]}, \quad n \geq 3,
$$

in the family $K$ of all continua $E$ of unit capacity. Schiffer and Goluzin studied this problem with the help of the variational method and obtained a differential equation for the extremal continuum. Later, Reich and Schiffer [RS] showed that each extremal continuum of the problem on $d_{n}$ satisfies this equation. The indicated result is stated as follows. 
Theorem XIV. Suppose that $n \geq 3, E$ is the extremal continuum of the problem pertaining to $d_{n}$, and $c_{1}, \ldots, c_{n}$ are the Fekete points on $E$. The continuum $E$ is the union of the closures of critical trajectories of the quadratic differential

$$
Q(z) d z^{2}=-\frac{2}{n(n-1)} \sum_{1 \leq k<l \leq n} \frac{d z^{2}}{\left(z-c_{k}\right)\left(z-c_{l}\right)} .
$$

We let $\zeta=g(z)$ be a conformal homeomorphism of the domain $D=\overline{\mathbb{C}} \backslash E$ onto $U^{*}$ with $g(\infty)=\infty$. The function $\zeta=g(z)$ satisfies in $D$ the equation

$$
\frac{d \zeta^{2}}{\zeta^{2}}=-Q(z) d z^{2}
$$

Clearly, the extremal continuum of the problem on $d_{n}$ is the smallest capacity continuum for its Fekete points: $E=E\left(c_{1}, \ldots, c_{n}\right)$.

Theorem XIV has led to a solution of the problem for $n=3$.

Theorem XV. In the family $K$, we have

$$
d_{3}^{3}=12 \sqrt{3} .
$$

Each extremal continuum $E$ consists of three segments of length $4^{1 / 3}$ emanating from one point at equal angles to each other.

As a consequence of Theorem XV, Goluzin obtained the following fact.

Theorem XVI. If $f(z) \in S$, then for any three points $c_{1}, c_{2}$, and $c_{3}$ that lie on three rays emanating from the origin at equal angles to each other and belong to the boundary of the domain $f(U)$, we have the inequality

$$
\prod_{k=1}^{3}\left|f\left(c_{k}\right)\right| \geq \frac{1}{4}
$$

Equality occurs only for the function $f(z)=z\left(1+\epsilon z^{3}\right)^{-2 / 3},|\epsilon|=1$.

This result by Goluzin has had a large number of continuations.

The problem concerning $d_{n}$ is an example of a problem where the associated quadratic differential has free poles. These problems present a significant difficulty, and only a few extremal problems with four and five free poles have been solved completely.

The problem on $d_{4}$ was solved only in 1980 (see Kuz1). The proof turned out to be complicated and required a combination of various methods of geometric function theory. For each $n \geq 3$, equation (12) is fulfilled for the mapping $z=f_{n}(\zeta)$ of the domain $U^{*}$ onto the exterior of the symmetric continuum $E_{n}^{*}=\left\{z: z^{n} \in[0,4]\right\}$, as well as for other mappings. In contrast to the case where $n=3$, the continuum $E_{4}^{*}$ does not realize the required maximum. The extremal continuum of the problem on $d_{4}$ consists of five analytic arcs, is symmetric with respect to both coordinate axes, and is quite simply described via the smallest capacity continuum $E(\alpha)=E\left(0, e^{i \alpha}, e^{-i \alpha}\right)$. We denote cap $E(\alpha)$ by $H(\alpha)$, and we denote by $c(\alpha)$ the zero of the associated quadratic differential determining the continuum $E(\alpha)$.

Theorem XVII. The maximum $d_{4}(E)$ in the family $K$ is realized by the continuum

$$
E_{4}=\left\{z: H\left(\alpha_{0}\right) z^{2} \in E\left(0, e^{i \alpha_{0}}, e^{-i \alpha_{0}}\right)\right\},
$$

where $\alpha_{0}$ is a solution of the equation $c(\alpha)=\frac{1}{3} \cos \alpha\left(\alpha_{0}=\pi / 2-0.1574 \ldots\right)$, whence

$$
d_{4}^{6}=16 H^{3}\left(\alpha_{0}\right) \sin ^{2} \alpha_{0}=128.136 \ldots .
$$


We observe that the value $d_{4}^{6}\left(E_{4}^{*}\right)$ is only insignificantly smaller: $d_{4}^{6}\left(E_{4}^{*}\right)=128$.

For $n \geq 5$, the question concerning $d_{n}$ remains open at present. In this connection, G. Schober conjectured that for all $n$ the differential (12) has no zeros of order $k \geq$ 2. Schober's conjecture was proved in Leung's paper [Le] and in the joint paper by Leung and Schober [LeScho] (in both papers, with elimination of certain values of $k$ ) and independently by Kuznetsov $\mathrm{Ku}$ for all cases.

The problem of cap $E\left(a_{1}, \ldots, a_{n}\right)$ is a special case of a more general question on the maximum of the product

$$
\prod_{k=1}^{n} R^{\alpha_{k}^{2}}\left(D_{k}, a_{k}\right)
$$

in the family $\mathcal{D}\left(a_{1}, \ldots, a_{n}\right)$ of all systems of nonoverlapping simply connected domains $D_{1}, \ldots, D_{n}$ satisfying the condition $a_{k} \in D_{k}, k=1, \ldots, n$, where $a_{1}, \ldots, a_{n}$ is a fixed system of distinct points of $\mathbb{C}$ and $\alpha_{1}, \ldots, \alpha_{n}$ is a given system of positive reals. (As usual, $R\left(D_{k}, a_{k}\right)$ is the conformal radius of the domain $D_{k}$ with respect to the point $a_{k} \in D_{k}$.)

Lavrent'ev obtained the first result in this direction. To state it, we denote by $\mathcal{D}^{\prime}\left(a_{1}, \ldots, a_{n}\right)$ the subfamily of $\mathcal{D}\left(a_{1}, \ldots, a_{n}\right)$ such that $\infty \notin D_{k}$ for each $k=1, \ldots, n$.

Theorem XVIII. In the family $\mathcal{D}^{\prime}\left(a_{1}, a_{2}\right)$, we have the sharp inequality

$$
\prod_{k=1}^{2} R\left(D_{k}, a_{k}\right) \leq\left|a_{1}-a_{2}\right|^{2} .
$$

If $\left\{a_{1}, a_{2}\right\}=\{-1,1\}$, then equality in (14) occurs only in the case where the domains $D_{1}$ and $\mathrm{D}_{2}$ are two half-planes and the imaginary axis is their common boundary.

Using his variational method, Goluzin solved the problem for $n=3$ completely.

Theorem XIX. In the family $\mathcal{D}\left(a_{1}, a_{2}, a_{3}\right)$, we have the sharp inequality

$$
\prod_{k=1}^{3} R\left(D_{k}, a_{k}\right) \leq \frac{64}{81 \sqrt{3}}\left|\left(a_{1}-a_{2}\right)\left(a_{1}-a_{3}\right)\left(a_{2}-a_{3}\right)\right| .
$$

If $a_{1}, a_{2}$, and $a_{3}$ are three equidistant points on the unit circle $|z|=1$, then equality in (15) occurs only in the case where the domains $D_{1}, D_{2}$, and $D_{3}$ are bounded by rays emanating from the origin at equal angles to each other and containing $a_{1}, a_{2}$, and $a_{3}$ on their bisectors.

Using Goluzin's variational method, Kolbina K1, K2 found the maximum of the product (13) in the family $\mathcal{D}^{\prime}\left(a_{1}, a_{2}\right)$ for any $\alpha_{1}$ and $\alpha_{2}$, and in the family $\mathcal{D}\left(a_{1}, a_{2}, a_{3}\right)$ for any $\alpha_{1}, \alpha_{2}$, and $\alpha_{3}$. Other generalizations of inequalities (14) and (15) have also been obtained.

The problem on the maximum of the product (13) in the family $\mathcal{D}\left(a_{1}, \ldots, a_{n}\right)$ (it will be Problem $A_{n}$ below) belongs to the presently well-known range of extremal decomposition problems. Studies by Goluzin and Kolbina stimulated interest in these problems. See $\S 5$ for results of the modulus method in extremal decomposition problems, in particular, for information about the proof of the results in [K1, K2 by this method.

The problem of the maximum of the product

$$
\prod_{k=1}^{n} R\left(D_{k}, a_{k}\right)\left\{\prod_{1 \leq k<l \leq n}\left|a_{k}-a_{l}\right|\right\}^{-2 /[(n-1)]}
$$

in the family $\mathcal{D}$ of all systems of nonoverlapping simply connected domains $D_{1}, \ldots, D_{n}$ satisfying the condition $a_{k} \in D_{k}, k=1, \ldots, n$, where $a_{1}, \ldots, a_{n}$ is a fixed system of distinct points of $\mathbb{C}$, will be called Problem $B_{n}$. The quantity (16) is invariant with 
respect to the group of linear-fractional automorphisms of $\overline{\mathbb{C}}$. For $n \geq 4$, Problem $B_{n}$ is a problem where the associated quadratic differential has free poles.

If $n=3$, then Problem $A_{n}$ for equal values of the parameters $\alpha_{k}$ coincides with Problem $B_{n}$, while for $n \geq 4$ these problems are distinct. The extremal configuration of Problem $B_{n}$ admits the following geometric characterization.

Theorem XX. Suppose $a_{1}, \ldots, a_{n}$ is an extremal system of points, and let $D_{1}, \ldots, D_{n}$, where $a_{k} \in D_{k}, k=1, \ldots, n$, be an extremal system of domains of Problem $B_{n}(n \geq 3)$. Then $\overline{\mathbb{C}} \backslash \prod_{k=1}^{n} D_{k}$ is the union of the closures of critical trajectories of the quadratic differential

$$
-\left\{\sum_{k=1}^{n}\left(z-a_{k}\right)^{-2}-\frac{2}{(n-1)} \sum_{1 \leq k<l \leq n}\left[\left(z-a_{k}\right)\left(z-a_{l}\right)\right]^{-1}\right\} d z^{2}
$$

This theorem was first obtained by Bakhtina $[\mathrm{B}$, who used the boundary variational method; another proof was presented in [F1]. Much as the differential (12), the differential (17) has no multiple zeros (see $[\mathrm{Ku}]$ ).

Problem $A_{4}$ for any value of the cross ratio of the four points $a_{k}$ and Problem $B_{4}$ were solved in Kuz2, and another solution of Problem $B_{4}$ belongs to Fedorov [F1]. We denote the quantity $(16)$ by $I\left(a_{1}, \ldots, a_{n}\right)$.

Theorem XXI. Suppose $a \in \mathbb{C} \backslash\{1,-1\}$. Then in the family $\mathcal{D}(-1,1, a, \infty)$ we have the following sharp inequalities:

$$
I(-1,1, a, \infty) \leq 4^{-10 / 3}\left|1-a^{2}\right|^{4 / 3} \operatorname{cap}^{-4} E(-1,1, a) \leq 4^{-8 / 3} 3^{2} .
$$

In the first estimate, equality occurs only for a quadruple of domains $D_{1}, \ldots, D_{4}$ such that the mapping $w=G\left(2(z-a) /\left(z^{2}-1\right)\right)$, where $G(t)=\frac{1}{2}(t+1 / t)$ is the Zhukovskiu function, maps each of them to the exterior of the smallest capacity continuum $E(-1,1, a)$. In the second estimate, equality occurs only in the case where the points $-1,1$, and a form a regular triangle: $a= \pm i \sqrt{3}$.

Problem $B_{5}$ was solved in [D5, Kuz3] under the additional assumption that the quintuple $a_{1}, \ldots, a_{5}$ is symmetric with respect to a line or a circle. No other ultimate results in Problems $A_{n}$ and $B_{n}$ for $n \geq 5$ are known at present.

Because of difficulty in solving Problem $B_{n}$ for $n \geq 5$, the problem was considered in the general case under additional symmetry conditions. The first results in this direction belong to Dubinin [D4]. He proved the following theorem.

Theorem XXII. Suppose $a_{1}, \ldots, a_{n}(n \geq 2)$ are points of the unit circle $|z|=1$. In the family $\mathcal{D}\left(a_{1}, \ldots, a_{n}\right)$, we have the sharp inequality

$$
\prod_{k=1}^{n} R\left(D_{k}, a_{k}\right) \leq(4 / n)^{n}
$$

Equality in (18) occurs only in the case where the $a_{k}$ are equidistributed points of the unit circle $|z|=1$, and the domains $D_{k}$ are angles formed by rays that emanate from the origin at equal angles to each other and contain the points $a_{k}$ on their bisectors.

There are other results pertaining to the same range of questions (see [D4, Kuz5]). While the method of separating transformation of domains was used in D4 (the method is discussed in $\S 5$ ), simple arguments of the extremal metric method were used in Kuz5. 


\section{§5. GEOMETRIC FUnCTION THEORY NOWADAYS}

5.1. In the years that followed, new methods arose in geometric function theory, and the previous classical methods were significantly developed. In the beginning of the 1950s, the method of the extremal metric entered geometric function theory. Of all the methods of geometric function theory, this method is most closely related to differential geometry and topology. The method involves the notion of extremal length of a family of curves (this notion, which was introduced by L. V. Ahlfors and A. C.-A. Beurling, generalizes Grötzsch's modulus significantly), Jenkins' extension of this notion to the case of several families of curves, and H. Renggli's proof of the uniqueness of the extremal metric in the modulus problem.

A general result of the extremal metric method is Jenkins' "general coefficient theorem". The theorem realizes Teichmüller's principle for a wide range of extremal problems and is the central subject of Jenkins' monograph J4. Using this theorem, Jenkins deduced a large number of the relatively elementary results of geometric function theory. However, the possibilities of the "fundamental coefficient theorem" are restricted by the normalizing.

In modern studies, it has become usual to widely employ the form of the extremal metric method that received the name of the method of moduli of families of curves (the modulus method for short). The method directly involves the relationship between the extremal metric problem for the family $\mathcal{H}$ of homotopy classes of curves and the extremal decomposition problem in an admissible family of systems of domains associated with the family $\mathcal{H}$. Extremal decomposition problems concern the maximum of a functional which is a linear combination of functions of domains - conformal invariants or their analogs, such as various reduced moduli-over an admissible family of systems of domains. Jenkins J3-1] was the first to consider extremal decomposition problems. The papers by P. M. Tamrazov, U. Pirl, and E. Renelt are among the first studies in the indicated range of questions.

We recall the definitions of the main functions of domains that are used below. The moduli of a doubly connected domain and of a quadrangle are defined in $\S 1$ of the present survey. The reduced modulus of a simply connected domain $D$ with respect to a point $a \in D$ is defined to be $M(D, a)=\frac{1}{2 \pi} \log R(D, a)$ if $a \in \mathbb{C}$, and we set $M(D, \infty)=-\frac{1}{2 \pi} \log R(D, \infty)$. (As before, $R(D, a)$ is the conformal radius of $D$ with respect to $a$.)

Suppose $D$ is a simply connected domain of hyperbolic type with distinguished boundary elements $a_{1}$ and $a_{2}$ supported at (distinct or coinciding) points $a_{1}$ and $a_{2}$. For the sake of definiteness, we assume that $a_{1}, a_{2} \in \mathbb{C}$. We also assume that $D$ satisfies the following condition: $(*)$ if $\zeta=g(z)$ is a conformal homeomorphism of $D$ onto the strip $-h / 2<\operatorname{Im} \zeta<h / 2$, and $\epsilon_{1}$ and $\epsilon_{2}$ are two sufficiently small positive reals, then in the connected component $\Delta_{k}\left(\epsilon_{k}\right)$ of $D \cap\left\{z:\left|z-a_{k}\right|<\epsilon_{k}\right\}$ containing $a_{k}$ as a boundary element we have the identity

$$
g(z)=(-1)^{k-1}\left\{A_{k} \log \left(z-a_{k}\right)+g_{k}(z)\right\}, \quad k=1,2,
$$

where $A_{k}>0$, and $g_{k}(z)$ is a regular function. (Clearly, $\varphi_{k}=h / A_{k}$ is the inner angle of the domain $D$ at the boundary element $a_{k}$.)

Suppose that $D$ satisfies condition $(*)$. We denote by $\Gamma$ the class of locally rectifiable curves in $D$ that join the sides of $D$. We denote by $S_{k}\left(\epsilon_{k}\right)$ the arc of the circle $\left|z-a_{k}\right|=\epsilon_{k}$ lying on the boundary of the domain $\Delta_{k}\left(\epsilon_{k}\right)$. Suppose $D\left(\epsilon_{1}, \epsilon_{2}\right) \subset D$ is a quadrangle with opposite sides $S_{k}\left(\epsilon_{k}\right), k=1,2$. We denote by $\Gamma\left(\epsilon_{1}, \epsilon_{2}\right)$ the class of locally rectifiable curves in $D\left(\epsilon_{1}, \epsilon_{2}\right)$ separating the sides $S_{1}\left(\epsilon_{1}\right)$ and $S_{2}\left(\epsilon_{2}\right)$, and we denote by $M^{(2)}\left(D\left(\epsilon_{1}, \epsilon_{2}\right)\right)$ the modulus of the quadrangle $D\left(\epsilon_{1}, \epsilon_{2}\right)$ with respect to the 
class $\Gamma\left(\epsilon_{1}, \epsilon_{2}\right)$. The limit

$$
M^{(2)}\left(D, a_{1}, a_{2}\right)=\lim _{\epsilon_{1}, \epsilon_{2} \rightarrow \infty}\left\{M^{(2)}\left(D\left(\epsilon_{1}, \epsilon_{2}\right)+\sum_{k=1}^{2} \varphi_{k}^{-1} \log \epsilon_{k}\right)\right\}
$$

is called the reduced modulus of the biangle $D$ for the class $\Gamma$ (or the modulus- 2 of the biangle $D$ ) with respect to the distinguished boundary elements $a_{1}$ and $a_{2}$ of $D$.

5.2. General results in the theory of the modulus method are known at present. In order to formulate the theorem on extremal decomposition under sufficiently general assumptions, we need several definitions.

Assume that $S=\overline{\mathbb{C}}$ or $S$ is a finitely connected domain on $\overline{\mathbb{C}}$ with nondegenerate boundary components. Suppose that

$$
A=\left\{a_{k}\right\}_{k=1}^{n}, \quad B=\left\{b_{l}\right\}_{l=1}^{m}, \quad \text { and } \quad B^{(1)}=\left\{b_{k}^{(1)}\right\}_{k=1}^{r}
$$

are sets of distinct points of $\bar{S}=S \cup \partial S$. One or two of these sets may be empty. We put

$$
S^{\prime}=S \backslash\left\{A \cup B \cup B^{(1)}\right\} .
$$

We consider the following four types of homotopy classes of locally rectifiable Jordan curves on $S^{\prime}$. The first type consists of the classes of closed curves that are not contractible on $S$. If $S \neq \overline{\mathbb{C}}$, then the classes of the second type consist of $\operatorname{arcs}$ on $S^{\prime}$ that join boundary elements of $S$. The third type consists of the classes of closed curves homotopic to a pointwise curve at $b_{l}$. Finally, if $B^{(1)} \neq \varnothing$, then the classes of the fourth type constitute the classes $H_{s}^{(1)}, s=1, \ldots, p$, of arcs on $S^{\prime}$ with endpoints at distinct or coinciding points $b_{k^{\prime}(s)}, b_{k^{\prime \prime}(s)} \in B^{(1)}$. We assume that each point $b_{k}^{(1)}$ is the endpoint of an arc belonging to one or several of the classes of the fourth type.

A free family $\mathcal{H}$ of homotopy classes of curves on $S^{\prime}$ is a set of classes $H_{i}, i=1, \ldots$, $j+j_{1}+m+p$, where the class $H_{i}$ has the first type for $i=1, \ldots, j$, the second type for $i=j+1, \ldots, j+j_{1}$, the third type for $i=j+j_{1}+1, \ldots, j+j_{1}+m$, and the fourth type for $i=j+j_{1}+m, \ldots, j+j_{1}+m+p$. Classes of one, two, or three of these types may be absent in $\mathcal{H}$. We assume that the classes $H_{i}$ are nonempty and pairwise distinct.

A doubly connected domain $D$ on $S^{\prime}$ is said to be associated with a class $H$ of the first type if the family of closed curves in $D$ separating the boundary components of $D$ is contained in $H$.

Suppose that $S \neq \overline{\mathbb{C}}$. Then a quadrangle $D$ on $S^{\prime}$ with opposite sides on the boundary of $S$ is said to be associated with a class $H$ of the second type if the family of arcs lying in $D$ and joining the sides of $D$ on $\partial S$ is contained in $H$. In each of the preceding two cases, by the modulus of the domain $D$ we mean the modulus of $D$ with respect to the family of closed curves or arcs in $D$ contained in the class $H$.

A simply connected domain $D$ on $S^{\prime} \cup\left\{b_{l}\right\}$ containing the point $b_{l}$ is said to be associated with a class $H$ of the third type, and by the modulus of $D$ we mean the reduced modulus $M\left(D, b_{l}\right)$ of $D$ with respect to the point $b_{l}$.

A biangle $D$ on $S^{\prime}$ with vertices at distinguished points of the set $B^{(1)}$ is said to be associated with a class $H$ of the fourth type if the $\operatorname{arcs}$ in $D$ joining the vertices of $D$ are contained in $H$. We assume that the biangle $D$ satisfies condition (*) (see Subsection 5.1). Then by the modulus of the biangle $D$ we mean the reduced modulus-2 of $D$ with respect to the vertices of $D$ (see the definition in Subsection 5.1).

We assume that the classes $H_{i}, i=1, \ldots, j+j_{1}+m+s$, are determined by the point systems $A, B$, and $B^{(1)}$ so that if a domain $D$ is associated with one of the classes $H_{i}$ (say, with a class $H$ ) of the first, second, or third type (of the fourth type), then the 
modulus of $D$ associated with the class $H$ is bounded from above (from below) by a quantity independent of the choice of $D$.

A finite number of nonoverlapping domains on $S^{\prime} \cup B$ are said to form an admissible system of domains $D_{i}$ associated with a family $\mathcal{H}$ of classes $H_{i}, i=1, \ldots, j+j_{1}+m+p$, if each of the domains is associated with a class $H_{i}$, and no two domains are associated with one and the same class $H_{i}$. If no domains are associated with a certain class $H_{i}$ of the first or the second type, then the corresponding domain $D_{i}$ is said to be degenerate, and the modulus of $D_{i}$ associated with the class $H_{i}$ is 0 by definition. We denote by $\mathcal{D}_{S}$ the family of all admissible systems of domains $D_{i}, i=1, \ldots, j+j_{1}+m+p$, associated with the family $\mathcal{H}$.

Suppose we are given two systems of positive reals

and set

$$
\boldsymbol{\alpha}=\left\{\alpha_{i}\right\}_{i=1}^{j+j_{1}+m} \text { and } \mathbf{h}=\left\{h_{s}\right\}_{s=1}^{p},
$$

$$
\alpha_{k}^{(1)}=\sum_{s \in I_{k}} h_{s}
$$

where $I_{k}$ is the set of the subscripts $s \in\{1, \ldots, p\}$ such that $b_{k}^{(1)}$ is an endpoint of the arcs in the class $H_{j+j_{1}+m+s}:=H_{s}^{(1)}$. (If the point $b_{k}^{(1)}$ is the endpoint in both directions for arcs in a certain class $H_{s}^{(1)}$, then the corresponding subscript $s$ occurs in $I_{k}$ twice.) We assume that the inner angle $\varphi_{k}$ of the biangle $D_{s}^{(1)}, s=1, \ldots, p$, at the vertex $b_{k}^{(1)}$ satisfies the condition

$$
\varphi_{k}=2 \pi h_{k} / \alpha_{k}^{(1)}
$$

We denote by $\mathcal{D}_{S}(\mathbf{h})$ the family of all systems of domains in $D_{S}$ satisfying this condition.

To simplify the formulations, we assume that $S=\overline{\mathbb{C}}$. In the sequel, $n+2(m+r) \geq r$, and the case where either $A=B^{(1)}=\varnothing$ and $m=2$ or $A=B=\varnothing$ and $r=2$ is excluded from the consideration.

We have the following theorem on extremal decomposition in the family $\mathcal{D}_{\overline{\mathbb{C}}}(\mathbf{h})$.

Theorem XXIII. Let $\mathcal{H}$ be the family defined above of homotopy classes of curves on $\overline{\mathbb{C}}^{\prime}=\overline{\mathbb{C}} \backslash\left\{A \cup B \cup B^{(1)}\right\}$, and let $\boldsymbol{\alpha}$ and $\mathbf{h}$ be two arbitrary systems of positive reals. There exists a quadratic differential on $\overline{\mathbb{C}}$ of the form

$$
Q(z) d z^{2}=P(z)\left\{\prod_{k=1}^{n}\left(z-a_{k}\right) \prod_{l=1}^{m}\left(z-b_{l}\right)^{2} \prod_{k=1}^{r}\left(z-b_{k}^{(1)}\right)^{2}\right\}^{-1} d z^{2},
$$

where $P(z)$ is a polynomial of degree at most $n+2(m+r)-4$ uniquely determined by the following conditions. We denote by $\Phi$ the union of all critical trajectories of the differential (19), and we denote by $\bar{\Phi}$ the closure of $\Phi$. The interior $\widehat{\Phi}$ of $\bar{\Phi}$ is empty, and we have $\overline{\mathbb{C}} \backslash \bar{\Phi}=\sum_{i=1}^{j+m+s} D_{i}^{*}$, where $D_{i}^{*}, i=1, \ldots, j+m+p$, is a system of domains in the family $D_{\overline{\mathbb{C}}}(\mathbf{h})$. We let $\zeta=g_{i}(z)$ (respectively, $\zeta=g_{j+l}(z)$ and $\zeta=g_{j+m+s}(z)$ ) be a conformal homeomorphism of the doubly connected domain $D_{i}^{*}, i=1, \ldots, j$, onto the circular annulus $1<|\zeta|<M_{i}$ (respectively, of the simply connected domain $D_{j+l}^{*}$, $l=1, \ldots, m$, onto the disk $|\zeta|<R_{l}$ with $g_{j+l}\left(b_{l}\right)=0$ and $g_{j+l}^{\prime}\left(b_{l}\right)=1$, and of the biangle $D_{j+m+s}^{*}, s=1, \ldots, p$, onto the strip $-1 / 2<\operatorname{Im} \zeta<1 / 2$ with $g_{j+m+s}\left(b_{k^{\prime}(s)}^{(1)}\right)=-\infty$ and $\left.g_{j+m+s}\left(b_{k^{\prime \prime}(s)}^{(1)}\right)=+\infty\right)$.

In the domain $D_{i}^{*}, i=1, \ldots, j+m$, we have the equation

$$
\alpha_{i}^{2} d \zeta^{2} / \zeta^{2}=-4 \pi^{2} Q(z) d z^{2},
$$

and in the biangle $D_{j+m+s}^{*}, s=1, \ldots, p$, we have the equation

$$
h_{s}^{2} d \zeta^{2}=Q(z) d z^{2} .
$$


The system of domains $D_{1}^{*}, \ldots, D_{j+m+p}^{*}$, and only it, realizes the maximum of the functional

$$
\begin{aligned}
F_{\overline{\mathbb{C}}}(\boldsymbol{\alpha}, \mathbf{h})= & \sum_{i=1}^{j} \alpha_{i}^{2} M\left(D_{i}\right)+\sum_{l=1}^{m} \alpha_{j+j_{1}+l}^{2} M\left(D_{j+j_{1}+l}, b_{l}\right) \\
& -\sum_{s=1}^{p} h_{s}^{2} M^{(2)}\left(D_{s}^{(1)}, b_{k^{\prime}(s)}^{(1)}, b_{k^{\prime \prime}(s)}^{(1)}\right)
\end{aligned}
$$

over the family $\mathcal{D}_{\overline{\mathbb{C}}}(\mathbf{h})$.

Theorem XXIII embraces various results on extremal decomposition problems as special cases.

We specifically mention two simplest particular cases of Theorem XXIII.

1) For $n \geq 2$, suppose that $A=\left\{a_{1}, \ldots, a_{n}\right\}$ is a system of points of $\mathbb{C}, B=\left\{b_{1}\right\}=$ $\{\infty\}, B^{(1)}=\varnothing$, and $\mathcal{H}=\left\{H_{1}\right\}$, where $H_{1}$ is the homotopy class of closed curves of the third type separating the point $b_{1}$ from points of $A$. Then Theorem XXIII coincides with Theorem XII: we have

$$
F_{\overline{\mathbb{C}}}(1,0)=M\left(D_{1}, \infty\right)=-\frac{1}{2 \pi} \log R\left(D_{1}, \infty\right)=-\frac{1}{2 \pi} \operatorname{cap} E\left(a_{1}, \ldots, a_{n}\right) .
$$

2 ) Suppose $B=\varnothing, m \geq 1$. Then equations (20) yield $m$ algebraic conditions for the parameters of the polynomial $P(z)$ in the expression (19):

$$
\alpha_{j+l}=2 \pi\left\{-Q_{l}\left(b_{l}\right)\right\}^{1 / 2}, \quad l=1, \ldots, m,
$$

where $Q_{l}(z)=\left(z-b_{l}\right)^{2} Q(z)$ if $b_{l} \neq \infty$, and $Q(z)=z^{-2}$ if $b_{l}=\infty$.

Therefore, the associated quadratic differential in the maximum problem for the product (13) over the families $\mathcal{D}^{\prime}\left(a_{1}, a_{2}\right)$ and $\mathcal{D}\left(a_{1}, a_{2}, a_{3}\right)$ is completely determined by the conditions of the problem, and the required maximum is found by direct integration of equations (20).

In the case where $S=\mathfrak{R}$ is a finite Riemann surface and $B=B^{(1)}=\varnothing$, the corresponding theorem on the extremal decomposition was proved in [J3-1]. In the case where $S=\overline{\mathbb{C}}$ and $B^{(1)}=\varnothing$, Theorem XXIII was presented in Kuz'mina's paper [Kuz1], where various applications were given. Jenkins [J3-2, Emel'yanov and Kuz'mina EKuz, and Solynin So3] extended Theorem XXIII to the case of a Riemann surface for arbitrary sets $A, B$, and $B^{(1)}$. We notice that in EKuz a more general case was also investigated where the family $H$ contains the classes of arcs asymptotically similar to logarithmic spirals of an arbitrarily prescribed slope. Strebel's monograph [St] is devoted to the role of quadratic differentials in extremal decomposition problems.

The modulus method has the advantage that the unknown parameters (functionals of the extremal problem under study) acquire precise geometric meaning: they turn out to be the singularities of the associated quadratic differential.

Solynin [So1 and Emel'yanov [E] studied the following question important for applications: how do the values of the functional investigated in an extremal decomposition problem depend on the choice of the distinguished points on a surface? In the case of the functional (21), we have the following result. We denote the functional (21) by $\mathcal{M}\left(\alpha_{i}\right)$, $\mathcal{M}\left(a_{k}\right)$, etc., thus emphasizing the fact that the values of the functional depend on the parameters indicated. We denote the differential (19) by $Q(z) d z^{2}$.

Theorem XXIV. 1) Suppose $\alpha_{i} \in \boldsymbol{\alpha}$ or $h_{s} \in \mathbf{h}$. Then

$$
\frac{\partial}{\partial \alpha_{i}} \mathcal{M}\left(\alpha_{i}\right)=2 \alpha_{i} M\left(D_{i}^{*}\right) \quad \text { and } \quad \frac{\partial}{\partial h_{s}} \mathcal{M}\left(h_{s}\right)=-2 h_{s} M\left(D_{j+m+s}^{*}, b_{k^{\prime}(s)}, b_{k^{\prime \prime}(s)}\right) .
$$


2) Suppose $a_{k} \in A, a_{k}=\infty$. Then

$$
\frac{\partial}{\partial a_{k}} \mathcal{M}\left(a_{k}\right)=\pi Q_{k}\left(a_{k}\right), \quad \text { where } Q_{k}(z)=\left(z-a_{k}\right) Q(z) .
$$

3) Suppose $b_{l} \in B \cup B^{(1)}, b_{l}=\infty$ (here, we have $\left.b_{m+k}=b_{k}^{(1)}\right)$. Then

$$
\frac{\partial}{\partial b_{l}} \mathcal{M}\left(b_{l}\right)=\pi Q_{l}^{\prime}\left(b_{l}\right), \quad \text { where } Q_{l}(z)=\left(z-b_{l}\right)^{2} Q(z) \text {. }
$$

In applications of the modulus method, one studies extremal decomposition problems, both in the plane of the independent variable and in the plane of images. Using invariance or simple transformation formulas for moduli of the domains $D_{i}^{*}$ of the extremal family in the plane of the independent variable and the fact that the images of the $D_{i}^{*}$ 's constitute an admissible family of domains in the corresponding extremal decomposition problem on the plane of images, for an appropriate choice of the constants $\alpha_{i}, h_{s}$ we obtain sharp inequalities characterizing the extremal configuration of the problem considered. Extremal decomposition problems are also of independent interest, and results pertaining to these problems were used in a number of studies.

The applicability of the results of the modulus method is limited by the fact that no final statements are known presently in those extremal decomposition problems where the associated quadratic differential has poles of order at least 3.

There are also other forms of the extremal metric method. At present, the method has found applications in various areas of mathematics and can be viewed as a general mathematical method (see [J6]).

5.3. The symmetrization method was a purely geometric method that had widely spread in geometric function theory in the beginning of the 1950s. The idea of symmetrization was first used in geometric problems. Various types of symmetrizing transformations are known, the simplest of them being the Steiner symmetrization, i.e., symmetrization with respect to a line, and Pólya-Szegö's circular symmetrization. More complicated types of symmetrization are the averaging symmetrization of sets and the radial-averaging symmetrization of a system of sets, which was introduced by M. M. Marcus and generalized by I. P. Mityuk and V. A. Shlyk.

Applications of the symmetrization method to function theory involve various principles of symmetrization showing that symmetrizing transformations do not increase certain characteristics of a domain. For example, the Pólya-Szegö symmetrization does not increase the Dirichlet integral. Therefore, it also does not increase the capacity of a condenser and the conformal radius of a domain.

After that, the symmetrization method was developed in two directions: general results in the theory of circular symmetrization were obtained, and new and more flexible symmetrization approaches were worked out. Baernstein started the first of these directions; for results in this direction see [D6. We briefly discuss the second direction. Original approaches have been worked out here and interesting applications have been obtained.

Using the method of polarization, Dubinin (see [D3]) obtained a simple solution of Gonchar's problem in potential theory. (The first proof was given by Tamrazov.) The problem is as follows: to find the minimum of the capacity of a condenser, the plates of which are collections of segments lying on the segment $[-1,1]$ under the condition that each plate has fixed total linear measure. Dubinin's solution of this problem raised great interest in the method of polarization.

Dubinin worked out a piecewise-separating transformation of condensers and domains. One of the first examples of employing this transformation is the solution of Hayman's 
problem on covering vertical segments in the class $S$. Consult, for instance, D4 for some subsequent applications. The separating transformation of condensers and domains is a general example of piecewise-separating symmetrization.

Quite a novel method in symmetrization theory is the so-called dissymmetrization method. In contrast to classical results, dissymmetrization does not increase the capacity of a condenser. Originally, Dubinin introduced the transformation of dissymmetrization when solving Gonchar's problem on the maximum of the harmonic measure of a simply connected domain obtained from the unit disk $U$ by deleting a system of radial segments of equal length with endpoints on the unit circle; see D1.

The method of polarization, the separating transformation of condensers and domains, and the dissymmetrization method are widely used in modern studies (see Dubinin's survey [D6]).

5.4. Along with the development of the extremal metric method and of the symmetrization method, the classical methods of geometric function theory have also been perfected. For example, a general and rather heuristical form of the area method was worked out. Lebedev's monograph [L3] is devoted to this method.

Solutions of difficult extremal problems involve a combination of various methods of geometric function theory. In many studies, the variational method, the extremal metric method, and the symmetrization method successfully complement each other. In many cases, the variational method automatically yields information about extremal functions. (This information can often be obtained directly from Teichmüller's principle.) However, this information, generally speaking, turns out to be insufficient for determining the extremal configuration. In some cases, the symmetrization method allows one to establish a symmetry in the position of the poles of the associated quadratic differential and thus makes it possible to reduce the problem to a simpler one. The role of the modulus method in solutions of extremal problems was discussed above. In certain cases, the extremal metric method plays the role of a verification method, which allows one to establish that an admissible configuration indeed possesses the required extremal property.

Jenkins' papers devoted to the solution of Gronwall's problem (see [J1) and to questions of the boundary distortion (see [J2]) present a well-known example of the efficiency of combining various methods, more precisely, the extremal metric method and the symmetrization method. Another example is given by the proof of Theorem XVII above, which involves the variational method, the modulus method, and Grunsky's inequalities (see Theorem VII). Along with other arguments, the proof of Theorem XXI involves the modulus method and results of the symmetrization method.

In recent years, significant progress has been made in various questions of the theory of conformal mapping. For example, the recent joint paper by Solynin and Zalgaller [SoZ], is of an isoperimetric nature. They showed that the regular $n$-gon, and only it, has the smallest logarithmic capacity in the family of all $n$-gons with fixed area; Solynin So2 obtained a starting result in this direction. Studies in [SoZ, So2 yield solutions of isoperimetric problems posed in the Pólya-Szegö monograph $\mathrm{PoSz}$. The proofs in these papers involve geometric constructions and results on the problem of the extremal decomposition of an $n$-gon into the corresponding triangles.

Barnard, Pearce, and Solynin [BaSo, BaPSo1, BaPSo2] obtained a number of results on the minimal area problem in classes of conformal mappings. An essential role in the proofs is played by symmetrizing transformations and by a construction of local variations of a special form.

To a large extent, we are indebted to Gennadiǔ Mikhallovich Goluzin for the development of the methods of geometric function theory and for the modern state of this theory. 


\section{G. M. Goluzin's RESEARCH PAPERS}

[1929] On some estimates for the functions realizing univalent conformal mapping of the disk, Mat. Sb. 36 (1929), no. 1-2, 152-172. (Russian)

[1933] An extension of the Carleman formula and its application to the analytic continuation of functions (jointly with V. I. Krylov), Mat. Sb. 40 (1933), no. 2, 144-149. (Russian)

[1934] Solution of basic plane problems of mathematical physics for the case of the Laplace equation and multiply connected domains, bounded by the circles (method of functional equations), Mat. Sb. 41 (1934), no. 2, 246-276. (Russian)

[1934a] Solution of the space Dirichlet problem for the Laplace equation and the domains bounded by a finite number of spheres, Mat. Sb. 41 (1934), no. 2, 277-283. (Russian)

[1935] On the theory of univalent conformal mappings, Mat. Sb. 42 (1935), no. 2, 169-190. (Russian)

[1935a] Solution of the plane heat conductivity problem for multiply connected domains, bounded by circles, in the case of the presence of an isolating layer, Mat. Sb. 42 (1935), no. 2, 191-198. (Russian)

[1935b] On the majoration principle in function theory, Mat. Sb. 42 (1935), no. 6, 647-650. (Russian)

[1936] On distortion theorems in the theory of conformal mappings, Mat. Sb. (N.S.) 1 (43) (1936), no. 1, 127-135. (Russian)

[1936a] Sur la représentation conforme, Mat. Sb. (N.S.) 1 (43) (1936), no. 3, 273-282. (French)

[1936b] Sur les théorèmes de rotation dans la théorie des fonctions univalentes, Mat. Sb. (N.S) 1 (43) (1936), no. 3, 293-296. (French)

[1937] On distortion theorems for the conformal mapping of multiply connected domains, Mat. Sb. (N.S.) 2 (44) (1937), no. 1, 37-64. (Russian)

[1937a] Some covering theorems for the functions regular in the disk, Mat. Sb. (N.S.) 2 (44) (1937), no. 3, 617-619. (Russian)

[1937b] An addendum to the work "On distortion theorems in the theory of conformal mappings", Mat. Sb. (N.S.) 2 (44) (1937), no. 4, 685-688. (Russian)

[1937c] On conformal mapping of two connected domains, bounded by rectilinear and circle polygons, Conformal Mapping of Simply Connected and Multiply Connected Domains, "ONTI", MoscowLeningrad, 1937, pp. 90-97. (Russian)

[1937d] Conformal mapping of multiply connected domains on the plane with slits by the method of functional equations, Conformal Mapping of Simply Connected and Multiply Connected Domains, "ONTI", Moscow-Leningrad, 1937, pp. 98-110. (Russian)

[1938] Some coefficient estimates of univalent functions, Mat. Sb. (N.S.) 3 (45) (1938), no. 2, 321-330. (Russian)

[1938a] On the method of continuity in the theory of conformal mappings of multiply connected domains, Mat. Sb. (N.S.) 4 (46) (1938), no. 1, 3-8. (Russian)

[1939] Iteration processes for conformal mapping of multiply connected domains, Mat. Sb. (N.S.) 6 (48) (1939), no. 3, 377-382. (Russian) MR0001846 (1:306c)

[1939a] On limiting values of Cauchy integral, Uchen. Zap. Leningrad. Gos. Univ. no. 37 (1939), 43-47 (= Uchen. Zap. Leningrad. Gos. Univ. Mat. Ser. no. 6 (1939), 43-47). (Russian) MR0003212 $(2: 181 \mathrm{~d})$

[1939b] On complete systems of functions in the complex domain, Uchen. Zap. Leningrad. Gos. Univ. no. 37 (1939), 48-51 (= Uchen. Zap. Leningrad. Gos. Univ. Mat. Ser. no. 6 (1939), 48-51). (Russian) MR0003245 (2:188b)

[1939c] On the theory of univalent functions, Mat. Sb. (N.S.) 6 (48) (1939), no. 3, 383-388. (Russian) MR0001857 (1:308b)

[1939d] Interior problems of the theory of univalent functions, Uspekhi Mat. Nauk vyp. 6 (1939), 26-89. (Russian) MR0000290(1:49b)

[1940] On p-valent functions, Mat. Sb. (N.S.) 8 (50) (1940), no. 2, 277-284. (Russian) MR0003234 $(2: 185 \mathrm{~d})$

[1943] On coefficients of univalent functions, Mat. Sb. (N.S.) 12 (54) (1943), no. 1, 40-47. (Russian) MR0009052 (5:93e)

[1943a] On the theory of univalent functions, Mat. Sb. (N.S.) 12 (54) (1943), no. 1, 48-55. (Russian) MR0009053(5:93f)

[1943b] On the wing theory in plane-parallel flow of fluids, Mat. Sb. (N.S.) 12 (54) (1943), no. 1, 146-151. (Russian)

[1945] Some estimations of derivatives of bounded functions, Mat. Sb. (N.S.) 16 (58) (1945), no. 3, 295-306. (Russian) MR0013802 (7:202a)

[1946] On the theory of univalent functions, Mat. Sb. (N.S.) 18 (60) (1946), no. 1, 167-179. (Russian) MR0016132(7:515c) 
[1946a] On the problem of Carathéodory-Fejér and similar problems, Mat. Sb. (N.S.) 18 (60) (1946), no. 2, 213-226. (Russian) MR0016478(8:22i)

[1946b] Some properties of polynomials, Mat. Sb. (N.S.) 18 (60) (1946), no. 2, 227-236. (Russian) MR0016477 (8:22h)

[1946c] On the distortion theorems in the theory of conformal mappings, Mat. Sb. (N.S.) 18 (60) (1946), no. 3, 379-390. (Russian) MR0020634 (8:9574a)

[1946d] On the number of finite asymptotic values of integral functions of finite order, Mat. Sb. (N.S.) 18 (60) (1946), no. 4, 391-396. (Russian) MR0016479 (8:23a)

[1946e] On distortion theorems and coefficients of univalent functions, Mat. Sb. (N.S.) 19 (61) (1946), no. 2, 183-202. (Russian) MR0018753 (8:325d)

[1946f] Methods of variations in conformal mapping. I, Mat. Sb. (N.S.) 19 (60) (1946), no. 2, 203-236. (Russian) MR0018752 (8:325c)

[1946g] Estimates for analytic functions with bounded mean of the modulus, Trudy Mat. Inst. Steklov. 18 (1946), 3-18. (Russian) MR0020633 (8:573h)

[1946h] The variational method in conformal mapping, Nauchn. Bull. Leningrad. Gos. Univ. no. 9 (1946), 3-5. (Russian)

[1947] Method of variations in conformal mapping. II, Mat. Sb. (N.S.) 21 (63) (1947), no. 1, 83-117. (Russian) MR0023908 (9:421e)

[1947a] Method of variations in conformal mapping. III, Mat. Sb., (N.S.) 21 (63) (1947), no. 1, 119-132. (Russian) MR0023909 (9:421f)

[1948] Some covering theorems in the theory of analytic functions, Mat. Sb. (N.S.) 22 (64) (1948), no. 3, 353-372. (Russian) MR0027054 (10:241b)

[1948a] On the coefficients of univalent functions, Mat. Sb. (N.S.) 22 (64) (1948), no. 3, 337-380. (Russian) MR0026688 (10:186d)

[1948b] On distortion theorems and the coefficients of univalent functions, Mat. Sb. (N.S.) 23 (65) (1948), no. 3, 353-360. (Russian) MR0029431(10:602a)

[1949] On analytic functions with the bounded mean modulus, Uchen. Zap. Leningrad. Gos. Univ. no. 111 (1949), 120-125. (Russian)

[1949a] Variation deduction of distortion theorems for univalent functions, Uchen. Zap. Leningrad. Gos. Univ. no. 111 (1949), 126-134. (Russian)

[1949b] Some inequalities for analytic functions, Izv. Akad. Nauk Kazakh. SSR Ser. Mat. Mekh. vyp. 3 (1949), 101-105. (Russian) MR0045814 (13:639b)

[1949c] Some questions of the theory of univalent functions, Trudy Mat. Inst. Steklov. 27 (1949), 111 pp. (Russian) MR0042510(13:123a)

[1949d] On mean values, Mat. Sb. (N.S.) 25 (67) (1949), no. 2, 307-314. (Russian) MR0032738 $(11: 339 \mathrm{c})$

[1950] Some estimates for bounded functions, Mat. Sb. (N.S.) 26 (68) (1950), no. 1, 7-18. (Russian) MR0033351(11:426e)

[1950a] On typically real functions, Mat. Sb. (N.S.) 27 (69) (1950), no. 2, 201-218. (Russian) MR0039060 (12:490e)

[1951] On the theory of univalent functions, Mat. Sb. (N.S.) 28 (70) (1951), no. 2, 351-358. (Russian) MR0045815 (13:639c)

[1951a] On the theory of univalent functions, Mat. Sb. (N.S.) 29 (71) (1951), no. 1, 197-208. (Russian) MR0043208 (13:223a)

[1951b] On majorants of subordinate analytic functions. I, Mat. Sb. (N.S.) 29 (71) (1951), no. 1, 209-224. (Russian) MR0043209 (13:223b)

[1951c] On subordinate univalent functions, Trudy Mat. Inst. Steklov. 38 (1951), 68-71. (Russian) MR0046432(13:733c)

[1951d] Variational method in conformal mapping. IV, Mat. Sb. (N.S.) 29 (71) (1951), no. 2, 455-468. (Russian) MR0044641(13:454a)

[1951e] On the parametric representation of functions univalent in a ring, Mat. Sb. (N.S.) 29 (71) (1951), no. 2, 469-476. (Russian) MR0047780(13:930b)

[1951f] On majoration of subordinate analytic functions. II, Mat. Sb. (N.S.) 29 (71) (1951), no. 3, 593-602. (Russian) MR0044644 (13:454d)

[1951g] On the problem of coefficients of univalent functions, Dokl. Akad. Nauk SSSR 81 (1951), no. 5, 721-723. (Russian) MR0045213 (13:546c)

[1952] On a variational method in the theory of analytic functions, Uchen. Zap. Leningrad. Gos. Univ. no. 144 (1952), 85-101 (Uchen. Zap. Leningrad. Univ. Ser. Mat. Nauk no. 23 (1952), 85-101). (Russian) MR0077640(17:1070d) 
[1952a] Geometrical theory of functions of a complex variable, "GITTL", Moscow-Leningrad, 1952; 2nd ed., "Nauka", Moscow, 1966; German transl., VEB Deutscher Verlag Wiss., Berlin, 1957; English transl., Transl. Math. Monogr., vol. 26, Amer. Math. Soc., Providence, RI, 1969. MR0219714 (36:2793) MR0089896 (19:735e) MR0247039(40:308)

\section{REFERENCES}

[A] I. A. Aleksandrov, Parametric continuations in the theory of univalent functions, "Nauka", Moscow, 1976. (Russian) MR0480952 (58:1099)

[Ai] L. A. Aǐzenberg, Carleman's formulas in complex analysis, "Nauka", Novosibirsk, 1990; English transl., Math. Appl., vol. 244, Kluwer Acad. Publ. Group, Dordrecht, 1993. MR1089612 (92b:32007) MR:1256735 (95d:32004)

[AlKuzL] Yu. E. Alenitsin, G. V. Kuz'mina, and N. A. Lebedev, Methods and results in geometric function theory, G. M. Goluzin, Geometric Theory of Functions of a Complex Variable, 2nd ed., "Nauka", Moscow, 1966, pp. 532-626; English transl., Transl. Math. Monogr., vol. 26, Amer. Math. Soc., Providence, RI, 1969. MR0219714 (36:2793), MR0247039 (40:308)

[B] G. P. Bakhtina, Variational methods and quadratic differentials in the problems on nonoverlapping domains, Thesis, Kiev, 1975, 12 pp. (Russian)

[BaSo] R. W. Barnard and A. Yu. Solynin, Local variations and minimal area problem for Carathéodory functions, Indiana Univ. Math. J. 53 (2004), no. 1, 135-167. MR2048187 (2005a:30028)

[BaPSo1] R. W. Barnard, K. Pearce, and A. Yu. Solynin, An isoperimetric inequality for logarithmic capacity, Ann. Acad. Sci. Fenn. Math. 27 (2002), no. 2, 419-436. MR1922198 (2003g:30039)

[BaPSo2] _ Area, width, and logarithmic capacity of convex sets, Pacific J. Math. 212 (2003), no. 1, 13-23. MR2016565 (2004j:30041)

[BarKh] V. A. Bart and V. P. Khavin, Szegö-Kolmogorov-Kreı̆n theorems on a weighted trigonometric approximation, and Carleman-type formulas, Ukrain. Mat. Zh. 46 (1994), no. 1-2, 100-127; English transl., Ukrainian Math. J. 46 (1994), no. 1-2, 101-132. MR.1294817 (95k:42004)

[Br1] L. de Branges, A proof of the Bieberbach conjecture, LOMI Preprints, no. E-5-84, Leningrad, 1984. (English)

[Br2] A proof of the Bieberbach conjecture, Acta Math. 154 (1985), 137-152. MR772434 (86h:30026)

[D1] V. N. Dubinin, Change of harmonic measure in symmetrization, Mat. Sb. (N.S.) 124 (1984), no. 2, 272-279; English transl. in Math. USSR-Sb. 52 (1985). MR746071 (85j:30049)

[D3] , Transformation of functions and the Dirichlet principle, Mat. Zametki 38 (1985), no. 1, 49-55; English transl., Math. Notes 38 (1985), no. 1-2, 539-542. MR804180 (87j:31005)

[D4] A separating transformation of domains, and problems on extremal decomposition, Zap. Nauchn. Sem. Leningrad. Otdel. Mat. Inst. Steklov. (LOMI) 168 (1988), 48-66; English transl., J. Soviet Math. 53 (1991), no. 3, 252-263. MR982483 (90c:30006)

[D5] - On the maximum of one conformal invariant, Preprint, Akad. Nauk SSSR Dal'nevost. Otdel., Inst. Prikl. Mat., Vladivostok, 1990. (Russian)

[D6] Symmetrization in the geometric theory of functions of a complex variable, Uspekhi Mat. Nauk 49 (1994), no. 1, 3-76; English transl., Russian Math. Surveys 49 (1994), no. 1, 1-79. MR.1307130 (96b:30054)

[D7] Conformal mappings and inequalities for algebraic polynomials, Algebra i Analiz 13 (2001), no. 5, 16-43; English transl., St. Petersburg Math. J. 13 (2002), no. 5, 717-737. MR.1882862 (2003j:30010)

[Du] P. Duren, Univalent functions, Grundlehren Math. Wiss., vol. 259, Springer-Verlag, New York-Berlin, 1983. MR708494 (85j:30034)

[E] E. G. Emel'yanov, Some properties of the moduli of families of curves, Zap. Nauchn. Sem. Leningrad. Otdel. Mat. Inst. Steklov. (LOMI) 144 (1985), 72-82; English transl., J. Soviet Math. 38 (1987), no. 4, 2081-2090. MR787415 (87i:30040)

[EKuz] E. G. Emel'yanov and G. V. Kuz'mina, Theorems on extremal partitioning in a family of systems of domains of different types, Zap. Nauchn. Sem. S.-Peterburg. Otdel. Mat. Inst. Steklov. (POMI) 237 (1997), 74-104; English transl., J. Math. Sci. (New York) 95 (1999), no. 3, 2221-2239. MR1691285(2000c:30082)

[F1] S. I. Fedorov, On the maximum of a conformal invariant in a problem on nonoverlapping domains, Zap. Nauchn. Sem. Leningrad. Otdel. Mat. Inst. Steklov. (LOMI) 112 (1981), 172183; English transl. in J. Soviet Math. 25 (1984), no. 2. MR644003 (83e:30026) 
[F2] Chebotarev's variational problem in the theory of the capacity of plane sets, and covering theorems for univalent conformal mappings, Mat. Sb. (N.S.) 124 (1984), no. 1, 121139; English transl. in Math. USSR-Sb. 52 (1985), no. 1. MR743060 (85j:30006)

[FiPom] C. H. FitzGerald and Ch. Pommerenke, The de Branges theorem on univalent functions, Trans. Amer. Math. Soc. 290 (1985), no. 2, 683-690. MR792819 (87b:30023)

[FoKuz] O. M. Fomenko and G. V. Kuz'mina, The last 100 days of the Bieberbach conjecture, Math. Intelligencer 8 (1986), no. 1, 40-47. MR823219 (87h:01059)

[G] G. M. Goluzin, Geometrical theory of functions of a complex variable, "GITTL", MoscowLeningrad, 1952; 2nd ed., "Nauka", Moscow, 1966; German transl. of 1st ed., VEB Deutscher Verlag Wiss., Berlin, 1957; English transl. of 2nd ed., Transl. Math. Monogr., vol. 26, Amer. Math. Soc., Providence, RI, 1969. MR0219714(36:2793) MR0219714(36:2793) MR0089896 (19:735e) MR0247039(40:308)

[Gr] H. Grunsky, Lectures on theory of functions in multiply connected domains, Vandenhoeck and Ruprecht, Göttingen, 1978. MR0463413 (57:3365)

[H] W. K. Hayman, Multivalent functions, Cambridge Tracts in Math. and Math. Phys., No. 48, Cambridge Univ. Press, Cambridge, 1958; 2nd ed., Cambridge Tracts in Math., No. 110, 1994. MR1310776 (96f:30003) MR:1310776 (96f:30003)

[J1] J. A. Jenkins, On a problem of Gronwall, Ann. of Math. (2) 59 (1954), no. 3, 490-504. MR0061170 (15:786b)

[J2] Some theorems on boundary distortion, Trans. Amer. Math. Soc. 81 (1956), no. 2, 477-500. MR0076862(17:956b)

[J3-1] - On the existence of certain general extremal metrics, Ann. of Math. (2) 66 (1957), 440-453. MR0090648 (19:845g)

[J3-2] _ On the existence of certain general extremal metrics. II, Tohoku Math. J. (2) 45 (1993), no. 2, 249-257. MR:1215927 (94h:31002)

[J4] Univalent functions and conformal mappings, Ergeb. Math. Grenzgeb. (N. F.), Bd. 18, Springer-Verlag, Berlin, 1958; 2nd ed. corrected, 1965. MR0096806 (20:3288)

[J5] $\quad$ On certain geometrical problems associated with capacity, Math. Nachr. 39 (1969), no. 4-6, 349-356. MR0249594 (40:2837)

[J6] The method of extremal metric, The Bieberbach Conjecture (West Lafayette, Ind., 1985), Math. Surveys Monogr., vol. 21, Amer. Math. Soc., Providence, RI, 1986, pp. 95-104. MR.875234(87k:30036)

[K1] L. I. Kolbina, Some extremal problems in conformal mapping, Dokl. Akad. Nauk SSSR (N.S.) 84 (1952), no. 5, 865-868. (Russian) MR0048582 (14:35e)

[K2] Conformal mapping of the unit circle onto mutually nonoverlapping regions, Vestnik Leningrad. Univ. Ser. Mat. Fiz. Khim. 1955, vyp. 2, 37-43. (Russian) MR0070723 (17:26d)

[KrKuh] S. L. Krushkal' and R. Kyunau, Quasiconformal mappings-new methods and applications, "Nauka", Novosibirsk, 1984; German transl., Teubner Texts in Math., vol. 54, Teubner, Leipzig, 1983. MR.730760 (85k:30032b) MR0730760(85k:30032b)

$[\mathrm{Ku}] \quad$ V. O. Kuznetsov, Properties of associated quadratic differentials in some extremal problems, Zap. Nauchn. Sem. Leningrad. Otdel. Mat. Inst. Steklov. (LOMI) 168 (1988), 85-97; English transl., J. Soviet Math. 53 (1991), no. 3, 277-284. MR982485 (90h:30057)

[Kuz1] G. V. Kuz'mina, Moduli of families of curves and quadratic differentials, Trudy Mat. Inst. Steklov. 139 (1980), 241 pp.; English transl., Proc. Steklov Inst. Math. 1982, no. 1, 231 pp. MR612632(84j:30038a) MR0664708 (84j:30038b)

[Kuz2] On the problem of the maximum of the product of conformal radii of nonoverlapping domains, Zap. Nauchn. Sem. Leningrad. Otdel. Mat. Inst. Steklov. (LOMI) 100 (1980), 131145; English transl. in J. Soviet Math. 19 (1982), no. 6. MR599943 (82c:30033)

[Kuz3] _ On the extremal partitioning of the Riemann sphere, Zap. Nauchn. Sem. Leningrad. Otdel. Mat. Inst. Steklov. (LOMI) 185 (1990), 72-95; English transl., J. Soviet Math. 59 (1992), no. 6, 1180-1196. MR1097590 (92k:30010)

[Kuz4] Methods of geometric function theory. I, II, Algebra i Analiz 9 (1997), no. 3, 41-103; no. 5, 1-50; English transl., St. Petersburg Math. J. 9 (1998), no. 3, 455-507; no. 5, 889-930. MR.1604397 (99c:30047a) MR.1604397(99c:30047a)

[Kuz5] The extremal metric method in problems of maximizing the product of powers of conformal radii of nonoverlapping domains in the presence of free parameters, Zap. Nauchn. Sem. S.-Peterburg. Otdel. Mat. Inst. Steklov. (POMI) 302 (2003), 52-67; English transl., J. Math. Sci. (New York) 129 (2005), no. 3, 3843-3851. MR2023032 (2004m:30034) 
[L1] N. A. Lebedev, Majorizing region for the expression $I=\ln z^{\lambda} f^{\prime}(z)^{1-\lambda} f(z)^{-\lambda}$ in the class $S$, Vestnik Leningrad. Univ. Ser. Mat. Fiz. Khim. 1955, vyp. 3, 29-41. (Russian) MR0072213 $(17: 248 \mathrm{~b})$

[L2] - Some estimates for functions regular and univalent in a circle, Vestnik Leningrad. Univ. Ser. Mat. Fiz. Khim. 1955, vyp. 4, 3-21. (Russian) MR0074514 (17:599c)

[L3] - The area principle in the theory of univalent functions, "Nauka", Moscow, 1975. (Russian) MR0450540(56:8834)

[Le] Y. J. Leung, On the Nth diameter problem in the class $\Sigma$, Complex Variables Theory Appl. 9 (1987), no. 2-3, 227-239. MR923223 (89d:30011)

[LeScho] Y. J. Leung and G. Schober, The Nth diameter problem in the class $\Sigma$, J. Analyse Math. 48 (1987), 247-266. MR910011 (89d:30012)

$[\mathrm{M}] \quad$ I. M. Milin, Univalent functions and orthonormal systems, "Nauka", Moscow, 1971; English transl., Transl. Math. Monogr., vol. 49, Amer. Math. Soc., Providence, RI, 1977. MR0369684 (51:5916) MR0427620(55:651)

[P] J. R. Partington, Interpolation, identification, and sampling, London Math. Soc. Monogr. (N.S.), vol. 17, The Clarendon Press, Oxford Univ. Press, New York, 1997. MR1473224 (99m:41001)

[Pi] U. Pirl, Uber die geometrische Gestalt eines Extremalkontinuums aus der Theorie der konformen Abbildung, Math. Nachr. 39 (1969), no. 1-6, 297-312. MR0254223 (40:7432)

[PoSz] G. Pólya and G. Szegö, Isoperimetric inequalities in mathematical physics, Ann. of Math. Stud., No. 27, Princeton Univ. Press, Princeton, NJ, 1951. MR0043486 (13:270d)

[Pom1] Ch. Pommerenke, Über die Subordination analytischer Funktionen, J. Reine Angew. Math. 218 (1965), 159-173. MR0180669 (31:4900)

[Pom2] - On a variational method for univalent functions, Michigan Math. J. 17 (1970), no. 1, 1-3. MR0255792(41:452)

[Pom3] - Univalent functions. With a chapter on quadratic differentials by G. Jensen, Math. Lehrbucher, Bd. 25, Vandenhoeck and Ruprecht, Göttingen, 1975. MR0507768 (58:22526)

[RS] E. Reich and M. Schiffer, Estimates for the transfinite diameter of a continuum, Math. Z. 85 (1964), no. 1, 91-106. MR0174721 (30:4921)

[SchaSp] A. C. Schaeffer and D. C. Spencer, Coefficient regions for schlicht functions, Amer. Math. Soc. Colloq. Publ., vol. 35, Amer. Math. Soc., New York, 1950. MR0037908(12:326c)

[SSp] M. Schiffer and D. C. Spencer, Functionals of finite Riemann surfaces, Princeton Univ. Press, Princeton, NJ, 1954. MR0065652 (16:461g)

[So1] A. Yu. Solynin, The dependence on parameters of the modulus problem for families of several classes of curves, Zap. Nauchn. Sem. Leningrad. Otdel. Mat. Inst. Steklov. (LOMI) 144 (1985), 136-145; English transl., J. Soviet Math. 38 (1987), no. 4, 2131-2139. MR/787420|(86h:30042)

[So2] Solution of the Pólya-Szegö isoperimetric problem, Zap. Nauchn. Sem. Leningrad. Otdel. Mat. Inst. Steklov. (LOMI) 168 (1988), 140-153; English transl., J. Soviet Math. 53 (1991), no. 3, 311-320. MR.982489 (90h:30059)

[So3] Moduli and extremal metric problems, Algebra i Analiz 11 (1999), no. 1, 3-86; English transl., St. Petersburg Math. J. 11 (2000), no. 1, 1-65. MR1691080 (2001b:30058)

[SoZ] A. Yu. Solynin and V. A. Zalgaller, An isoperimetric inequality for logarithmic capacity of polygons, Ann. of Math. (2) 159 (2004), no. 1, 277-303. MR2052355 (2005a:31002)

[St] K. Strebel, Quadratic differentials, Ergeb. Math. Grenzgeb. (3), Bd. 5, Springer-Verlag, Berlin-New York, 1984. MR0743423 (86a:30072)

St. Petersburg Branch, Steklov Mathematical Institute, Russian Academy of Sciences, Fontanka 27, St. Petersburg 191023, Russia

E-mail address: kuzmina@pdmi.ras.ru

Received 30/MAR/2006

Translated by N. YU. NETSVETAEV 